\title{
Clipper: $p$-value-free FDR control on high-throughput data from two conditions
}

\author{
Xinzhou Ge ${ }^{1 \dagger}$, Yiling Elaine Chen ${ }^{1 \dagger}$, Dongyuan Song ${ }^{2}$, MeiLu McDermott ${ }^{3,4}$, Kyla Woyshner ${ }^{3}$, \\ Antigoni Manousopoulou ${ }^{3}$, Ning Wang ${ }^{2}$, Wei Li ${ }^{5}$, Leo D. Wang ${ }^{3}$ and Jingyi Jessica Li $\mathrm{Li}^{1,2,6,7,8^{*}}$ (1)
}

\author{
*Correspondence: jli@stat.ucla.edu \\ ${ }^{\dagger}$ Xinzhou Ge and Yiling Elaine Chen \\ contributed equally to this work. \\ 1 Department of Statistics, University \\ of California, Los Angeles, 90095 CA \\ USA \\ ${ }^{2}$ Interdepartmental Program in \\ Bioinformatics, University of \\ California, Los Angeles, 90095 CA \\ USA \\ Full list of author information is \\ available at the end of the article
}

\begin{abstract}
High-throughput biological data analysis commonly involves identifying features such as genes, genomic regions, and proteins, whose values differ between two conditions, from numerous features measured simultaneously. The most widely used criterion to ensure the analysis reliability is the false discovery rate (FDR), which is primarily controlled based on $p$-values. However, obtaining valid $p$-values relies on either reasonable assumptions of data distribution or large numbers of replicates under both conditions. Clipper is a general statistical framework for FDR control without relying on $p$-values or specific data distributions. Clipper outperforms existing methods for a broad range of applications in high-throughput data analysis.
\end{abstract}

\section{Introduction}

High-throughput technologies are widely used to measure system-wide biological features, such as genes, genomic regions, and proteins ("high-throughput" means the number of features is large, at least in thousands). The most common goal of analyzing high-throughput data is to contrast two conditions so as to reliably screen "interesting features," where "interesting" means "enriched" or "differential." "Enriched features" are defined to have higher expected measurements (without measurement errors) under the experimental (i.e., treatment) condition than the background (i.e., negative control) condition. The detection of enriched features is called "enrichment analysis." For example, typical enrichment analyses include calling protein-binding sites in a genome from chromatin immunoprecipitation sequencing (ChIP-seq) data [1,2] and identifying peptides from mass spectrometry (MS) data [3]. In contrast, "differential features" are defined to have different expected measurements between two conditions, and their detection is called "differential analysis." For example, popular differential analyses include the identification of differentially expressed genes (DEGs) from genome-wide gene expression data (e.g., microarray and RNA sequencing (RNA-seq) data [4-10]) and differentially interacting chromatin regions (DIRs) from Hi-C data [11-13] (Fig. 1a). In most scientific

(c) The Author(s). 2021 Open Access This article is licensed under a Creative Commons Attribution 4.0 International License, which permits use, sharing, adaptation, distribution and reproduction in any medium or format, as long as you give appropriate credit to the original author(s) and the source, provide a link to the Creative Commons licence, and indicate if changes were made. The images or other third party material in this article are included in the article's Creative Commons licence, unless indicated otherwise in a credit line to the material. If material is not included in the article's Creative Commons licence and your intended use is not permitted by statutory regulation or exceeds the permitted use, you will need to obtain permission directly from the copyright holder. To view a copy of this licence, visit http://creativecommons.org/licenses/by/4.0/. The Creative Commons Public Domain Dedication waiver (http://creativecommons.org/publicdomain/zero/1.0/) applies to the data made available in this article, unless otherwise stated in a credit line to the data. 
research, the interesting features only constitute a small proportion of all features, and the remaining majority is referred to as "uninteresting features."

The identified features, also called the "discoveries" from enrichment or differential analysis, are subject to further investigation and validation. Hence, to reduce experimental validation that is often laborious or expensive, researchers demand reliable discoveries that contain few false discoveries. Accordingly, the false discovery rate (FDR) [14] has been developed as a statistical criterion for ensuring discoveries' reliability. Technically, under the frequentist statistical paradigm, the FDR is defined as the expected proportion of uninteresting features among the discoveries. In parallel, under the Bayesian statistical paradigm, other criteria have been developed, including the Bayesian false discovery rate [15], the local false discovery rate (local fdr) [16], and the local false sign rate [17]. Among all these frequentist and Bayesian criteria, the FDR is the dominant criterion for setting thresholds in biological data analysis $[1,10,18-24]$ and is thus the focus of this paper.

FDR control refers to the goal of finding discoveries such that the FDR is under a pre-specified threshold (e.g., 0.05). Existing computational methods for FDR control primarily rely on $p$-values, one per feature. Among the $p$-value-based FDR control methods, the most classic and popular ones are the Benjamini-Hochberg $(\mathrm{BH})$ procedure [14] and the Storey's q-value [25]; later development introduced methods that incorporate feature weights [26] and/or covariates-e.g., independent hypothesis weighting (IHW) [27], adaptive $p$-value thresholding [28], and Boca and Leek's FDR regression [29] - to boost the detection power. All these methods set a $p$-value cutoff based on the pre-specified FDR threshold. However, the calculation of $p$-values requires either distributional assumptions, which are often questionable, or large numbers of replicates, which are often unachievable in biological studies (see the "Results" section). Due to the difficulty of valid $p$-value calculation in high-throughput biological data analysis, bioinformatics tools often output ill-posed $p$-values. This issue is evidenced by serious concerns about the widespread miscalculation and misuse of $p$-values in the scientific community [30]. As a result, bioinformatics tools using questionable $p$-values either cannot reliably control the FDR to a target level [23] or lack power to make discoveries [31]; see the "Results" section. Therefore, $p$-value-free control of FDR is desirable, as it would make data analysis more transparent and thus improve the reproducibility of scientific research.

Although $p$-value-free FDR control has been implemented in the MACS2 method for ChIP-seq peak calling [1] and the SAM method for microarray DEG identification [32], these two methods are restricted to specific applications and lack a theoretical guarantee for FDR control. (Although later works have studied some theoretical properties of SAM, they are not about the exact control of the FDR $[33,34]$.) More recently, the BarberCandès $(\mathrm{BC})$ procedure has been proposed to achieve theoretical FDR control without using $p$-values [35], and it has been shown to perform comparably to the $\mathrm{BH}$ procedure using well-calibrated $p$-values [36]. The BC procedure is advantageous because it does not require well-calibrated $p$-values, so it holds tremendous potential in various highthroughput data analyses where $p$-value calibration is challenging [37]. For example, a recent paper has implemented a generalization of the $\mathrm{BC}$ procedure to control the FDR in peptide identification from MS data [38].

Inspired by the $\mathrm{BC}$ procedure, we propose a general statistical framework Clipper to provide reliable FDR control for high-throughput biological data analysis, without using $p$-values or relying on specific data distributions. Clipper is a robust and flexible 


\section{a High-throughput omics data analyses}

Peak calling from ChIP-seq data

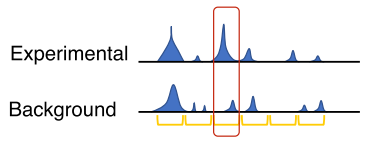

Peptide identification from MS data

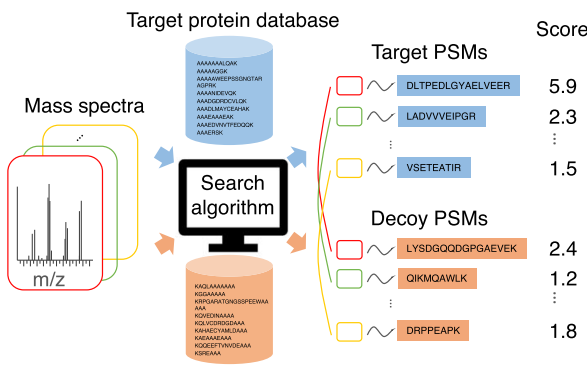

DEG analysis from RNA-seq data Condition 1 Genes 1 .

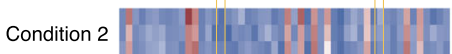

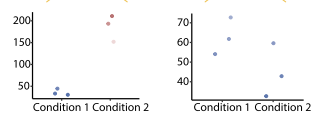

DIR analysis from $\mathrm{Hi}-\mathrm{C}$ data

Condition 1

Condition 2

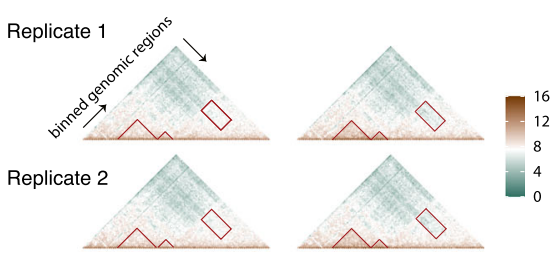

Decoy protein database

\section{b Generic FDR control methods}

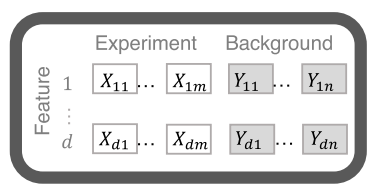

$\mathrm{BH} /$ qvalue-pair
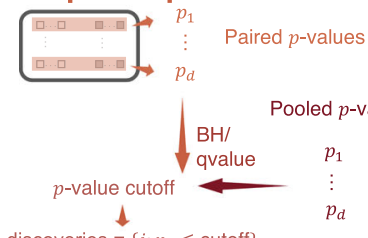

discoveries $=\left\{j: p_{j} \leq\right.$ cutoff $\}$

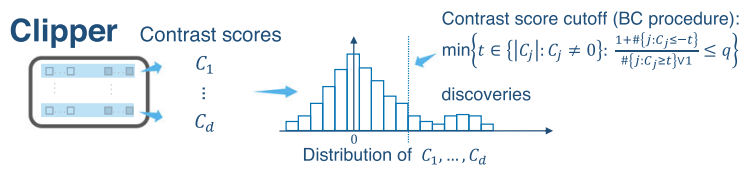

BH/qualue-pool
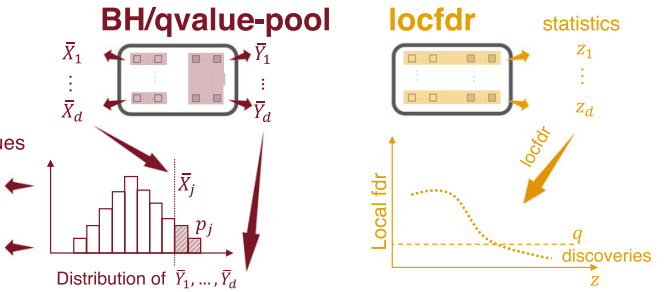

Fig. 1 High-throughput omics data analyses and generic FDR control methods. (a) Illustration of four common high-throughput omics data analyses: peak calling from ChIP-seq data, peptide identification from MS data, DEG analysis from RNA-seq data, and DIR analysis from $\mathrm{Hi}-\mathrm{C}$ data. In these four analyses, the corresponding features are genomic regions (yellow intervals), peptide-spectrum matches (PSMs; a pair of a mass spectrum and a peptide sequence), genes (columns in the heatmaps), and chromatin interacting regions (entries in the heatmaps). (b) Illustration of Clipper and five generic FDR control methods: BH-pair (and qvalue-pair), BH-pool (and qvalue-pool), and locfdr. The input data are $d$ features with $m$ and $n$ repeated measurements under the experimental and background conditions, respectively. Clipper computes a contrast score for each feature based on the feature's $m$ and $n$ measurements, decides a contrast-score cutoff, and calls the features with contrast scores above the cutoff as discoveries. (This illustration is Clipper for enrichment analysis with $m=n$.) BH-pair or qvalue-pair computes a $p$-value for each feature based on the feature's $m$ and $n$ measurements, sets a $p$-value cutoff, and calls the features with $p$-values below the cutoff as discoveries. BH-pool or qvalue-pool constructs a null distribution from the $d$ features' average (across the $n$ replicates) measurements under the background condition, calculates a $p$-value for each feature based on the null distribution and the feature's average (across the $m$ replicates) measurements under the experimental condition, sets a $p$-value cutoff, and calls the features with $p$-values below the cutoff as discoveries. The locfdr method computes a summary statistic for each feature based on the feature's $m$ and $n$ measurements, estimates the empirical null distribution and the empirical distribution of the statistic across features, computes a local fdr for each feature, sets a local fdr cutoff, and calls the features with local fdr below the cutoff as discoveries 
framework that applies to both enrichment and differential analyses and that works for high-throughput data with various characteristics, including data distributions, replicate numbers (from one to multiple), and outlier existence.

\section{Results}

Clipper consists of two main steps: construction and thresholding of contrast scores. First, Clipper defines a contrast score for each feature, as a replacement of a $p$-value, to summarize that feature's measurements between two conditions and to describe the degree of interestingness of that feature. Second, as its name suggests, Clipper establishes a cutoff on features' contrast scores and calls as discoveries the features whose contrast scores exceed the cutoff (see the "Methods" section and Additional File 1: Section S2). Clipper is a flexible framework that only requires a minimal input: all features' measurements under two conditions and a target FDR threshold (e.g., 5\%) (Fig. 1b).

Clipper only relies on two fundamental statistical assumptions of biological data analysis: (1) measurement errors (i.e., differences between measurements and their expectations, with the expectations including both biological signals and batch effects) are independent across all features and replicates and (2) every uninteresting feature has measurement errors identically distributed across all replicates under both conditions. These two assumptions are used in almost all bioinformatics tools and are commonly referred to as the "measurement model" in statistical genomics [39]. With these two assumptions, Clipper has a theoretical guarantee for FDR control under both enrichment and differential analyses with any number of replicates (see the "Methods" section and Additional File 1: Section S2).

To verify Clipper's performance, we designed comprehensive simulation studies to benchmark Clipper against existing generic FDR control methods (Additional File 1: Section S1). We also benchmarked Clipper against bioinformatics tools in studies including peak calling from ChIP-seq data, peptide identification from mass spectrometry data, DEG identification from bulk and single-cell RNA-seq data, and DIR identification from Hi-C data. Notably, our benchmarking results for peptide identification are based on our in-house data, the first MS data standard with a realistic dynamic range.

\section{Clipper has verified FDR control and power advantage in simulation}

Simulation is essential because we can generate numerous datasets from the same distribution with known truths to calculate the FDR, which is not observable from real data. Our simulation covers both enrichment and differential analyses. In enrichment analysis, we consider four "experimental designs": 1vs1 design (one replicate per condition), 2vs1 design (two and one replicates under the experimental and background conditions, respectively), 3vs3 design (three replicates per condition), and 10vs10 design (ten replicates per condition). In differential analysis, since Clipper requires that at least one condition has two replicates, we only consider the 2vs1 and 3vs3 designs. For each analysis and design, we simulated data from three "distributional families"-Gaussian, Poisson, and negative binomial-for individual features under two "background scenarios" (i.e., scenarios of the background condition): homogeneous and heterogeneous. Under the homogeneous scenario, all features' measurements follow the same distribution under the background condition; otherwise, we are under the heterogeneous scenario, which is ubiquitous in applications, e.g., identifying DEGs from RNA-seq data 
and calling protein-binding sites from ChIP-seq data. By simulation setting, we refer to a combination of an experimental design, a distributional family, and a background scenario. The details of simulation settings are described in Additional File 1: Section S4.

For both enrichment and differential analyses and each simulation setting, we compared Clipper against generic FDR control methods, including $p$-value-based methods and local-fdr-based methods. The $p$-value-based methods include BH-pair, BH-pool, qvalue-pair, and qvalue-pool, where "BH" and "qvalue" stand for $p$-value thresholding procedures, and "pair" and "pool" represent the paired and pooled $p$-value calculation approaches, respectively. The local-fdr-based methods include locfdr-emp and locfdrswap, where "emp" and "swap" represent the empirical null and swapping null local-fdr calculation approaches, respectively. See the "Methods" section for details.

The comparison results are shown in Fig. 2 and Additional File 1: Figures S1-S14. A good FDR control method should have its actual FDR no larger than the target FDR threshold and achieve high power. The results show that Clipper controls the FDR and is overall more powerful than the other methods, excluding those that fail to control the FDR, under all settings. Clipper is also shown to be more robust to the number of features and the existence of outliers than the other methods. In detail, in both enrichment analyses (1vs1, 2vs1, 3vs3, and 10vs10 designs) and differential analyses (2vs1 and 3vs3 designs), Clipper consistently controls the FDR, and it is more powerful than the generic methods in most cases under the realistic, heterogeneous background, where features do not follow the same distribution under the background condition. Under the idealistic, homogeneous background, Clipper is still powerful and only second to BH-pool and qvalue-pool, which, however, cannot control the FDR under the heterogeneous background.

Here we summarize the performance of the generic FDR control methods. First, the two p-value-based methods using the pooled approach-BH-pool and qvalue-pool-are the most powerful under the idealistic, homogeneous background, which is their inherent assumption; however, they cannot control the FDR under the heterogeneous background (Fig. 2b). Besides, they cannot control the FDR when the number of features is small (Fig. 2a and Additional File 1: Figure S1). These results show that the validity of BH-pool and qvalue-pool requires a large number of features and the homogeneous background assumption, two requirements that rarely both hold in biological applications.

Second, the four $p$-value-based methods using the paired approach with misspecified models or misformulated tests (BH-pair-mis, qvalue-pair-mis, BH-pair-2as1, and qvaluepair-2as1; see the "Methods" section) fail to control the FDR by a large margin in most cases, and rarely when they control the FDR, they lack power (Fig. 2c, $d$ and Additional File 1: Figures S1-S8). These results confirm that BH-pair and qvalue-pair rely on the correct model specification to control the FDR; however, the correct model specification is hardly achievable with no more than three replicates per condition.

Third, even when models are correctly specified (an idealistic scenario), the $p$-valuebased methods that use the paired approach-BH-pair-correct and qvalue-pair-correct (see the "Methods" section) - fail to control the FDR in the existence of outliers (Fig. 2e and Additional File 1: Figures S3 and S7) or for the negative binomial distribution with unknown dispersion (Fig. 2 f and Additional File 1: Figure S9). It is worth noting that even when they control the FDR, they are less powerful than Clipper in most cases except for the 3vs3 differential analysis with the Poisson distribution (Fig. 2d and Additional File 1: Figures S4 and S8). 
a
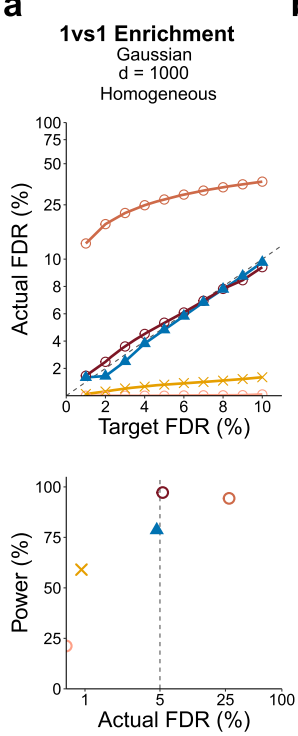

d
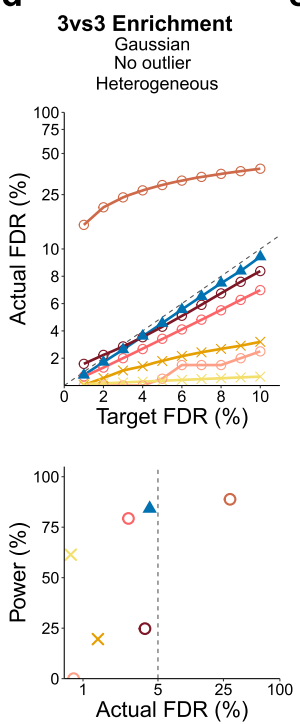

b
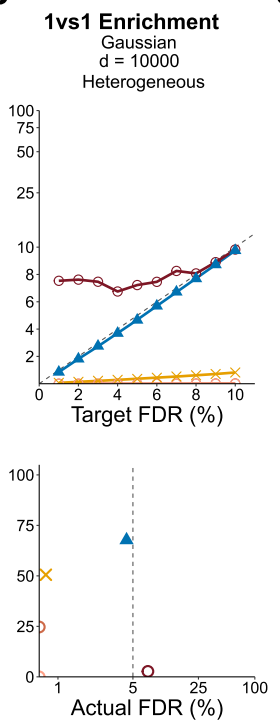

e

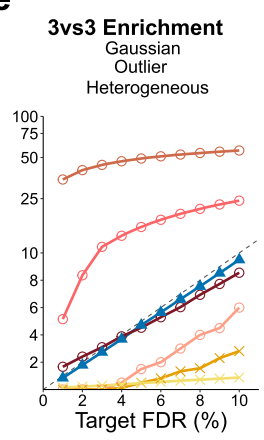

$\mathbf{f}$

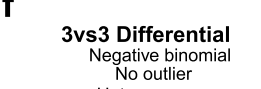

No outlier
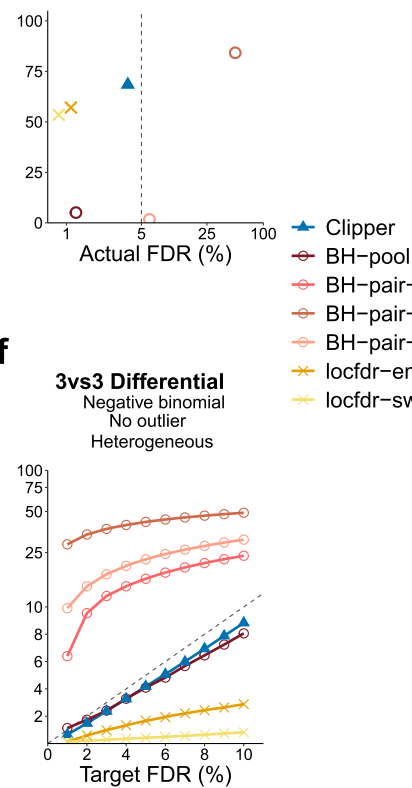

$\bullet \mathrm{BH}-$ pair-correct

- BH-pair-2as1

- BH-pair-mis

* locfdr-emp

* locfdr-swap
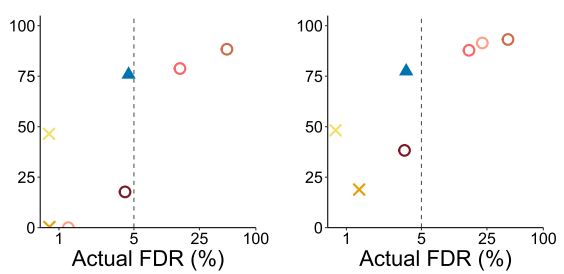

Fig. 2 Comparison of Clipper with generic FDR control methods in terms of their FDR control and power in six example simulation studies. a 1vs 1 enrichment analysis with 1000 features generated from the Gaussian distribution with a homogeneous background. b 1vs 1 enrichment analysis with 10,000 features generated from the Gaussian distribution with a heterogeneous background. c 2vs1 enrichment analysis with 10,000 features generated from the Poisson distribution with a heterogeneous background. d $3 \mathrm{vs} 3$ enrichment analysis with 10,000 features generated from the Gaussian distribution without outliers and with a heterogeneous background. e 3vs3 enrichment analysis with 10,000 features generated from the Gaussian distribution with outliers and with a heterogeneous background. $\mathbf{f} 3 \mathrm{vs} 3$ differential analysis with 10,000 features generated from the negative binomial distribution with a heterogeneous background. At target FDR thresholds $q \in\{1 \%, 2 \%, \cdots, 10 \%\}$, each method's actual FDRs and power are approximated by the averages of false discovery proportions (see Additional File 1: Eq. (S14)) and power evaluated on 200 simulated datasets. In each panel, the top row shows each method's actual FDRs at target FDR thresholds: whenever the actual FDR is larger than the target FDR (the solid line is higher than the dashed line), FDR control is failed; the bottom row shows each method's actual FDRs and power at the target FDR threshold $q=5 \%$ : whenever the actual FDR is greater than $q$ (on the right of the vertical dashed line), FDR control is failed. Under the FDR control, the larger the power, the better. Note that BH-pair-correct is not included in a-c because it is impossible to correctly specify the model with only one replicate per condition; locfdr-swap is not included in $\mathbf{a}$ and $\mathbf{b}$ because it is inapplicable to the 1vs1 design 
Fourth, the two local-fdr-based methods-locfdr-emp and locfdr-swap-achieve the FDR control for all designs and analyses; however, they are less powerful than Clipper in most cases (Additional File 1: Figures. S1-S4).

Fifth, when the numbers of replicates are large (10vs10 design), non-parametric tests become applicable. We compared Clipper with three BH-pair methods that use different statistical tests: BH-pair-Wilcoxon (the non-parametric Wilcoxon rank-sum test), BHpair-permutation (the non-parametric permutation test), and BH-pair-parametric (the parametric test based on the correct model specification, equivalent to BH-pair-correct). Although all the three methods control the FDR, they are less powerful than Clipper (Additional File 1: Figure S10).

Moreover, the above five phenomena are consistently observed across the three distributions (Gaussian, Poission, and negative binomial) that we have examined, further confirming the robustness of Clipper.

In addition, for the $3 \mathrm{vs} 3$ enrichment analysis, we also varied the proportion of interesting features as $10 \%, 20 \%$, and $40 \%$. The comparison results in Additional File 1: Figure S3 (columns 1 and 3 for 10\%) and Additional File 1: Figure S11 (for 20\% and 40\%) show that the performance of Clipper is robust to the proportion of interesting features.

The above results are all based on simulations with independent features. To examine the robustness of Clipper, we introduced feature correlations to our simulated data, on which we compared Clipper with the other generic FDR control methods. The comparison results in Additional File 1: Figure S12 show that even when the feature independence assumption is violated, Clipper still demonstrates strong performance in both FDR control and power.

\section{Clipper has broad applications in omics data analyses}

We then demonstrate the use of Clipper in four omics data applications: peak calling from ChIP-seq data, peptide identification from MS data, DEG identification from bulk or single-cell RNA-seq data, and DIR identification from Hi-C data. The first two applications are enrichment analyses, and the last two are differential analyses. In each application, we compared Clipper with mainstream bioinformatics methods to demonstrate Clipper's superiority in FDR control and detection power.

\section{Peak calling from ChIP-seq data (enrichment analysis I)}

ChIP-seq is a genome-wide experimental assay for measuring binding intensities of a DNA-associated protein [40], often a transcription factor that activates or represses gene expression [41, 42]. ChIP-seq data are crucial for studying gene expression regulation, and an indispensable analysis is to identify genomic regions with enriched sequence reads in ChIP-seq data. These regions are likely bound by the target protein and thus of biological interest. The identification of these regions is termed "peak calling" in ChIP-seq data analysis.

As the identified peaks are subject to experimental validation that is often expensive [43], it is essential to control the FDR of peak identification to reduce unnecessary costs. The two most highly-cited peak-calling methods are MACS2 [1] and HOMER [2], both of which claim to control the FDR for their identified peaks. Specifically, both MACS2 and HOMER assume that the read counts for each putative peak (with one count per sample/replicate) follow the Poisson distribution, and they use modified paired approaches 
to assign each putative peak a $p$-value and a corresponding Storey's $q$-value. Then given a target FDR threshold $0<q<1$, they call the putative peaks with $q$-values $\leq q$ as identified peaks. Despite being popular, MACS2 and HOMER have not been verified for their FDR control, to our knowledge.

To verify the FDR control of MACS2 and HOMER (Additional File 1: Section S5.1), we used ENCODE ChIP-seq data of cell line GM12878 [44] and ChiPulate [45], a ChIP-seq data simulator, to generate semi-synthetic data with spiked-in peaks (Additional File 1: Section S6.1). We examined the actual FDR and power of MACS2 and HOMER in a range of target FDR thresholds: $q=1 \%, 2 \%, \ldots, 10 \%$. Figure 3a shows that MACS2 and HOMER cannot control the FDR as standalone peak-calling methods. However, with Clipper as an add-on (Additional File 1: Section S7.1), both MACS2 and HOMER can guarantee the FDR control. This result demonstrates the flexibility and usability of Clipper for reducing false discoveries in peak calling analysis.

Technically, the failed FDR control by MACS2 and HOMER is attributable to the likely model misspecification and test misformulation in their use of the paired approach. Both

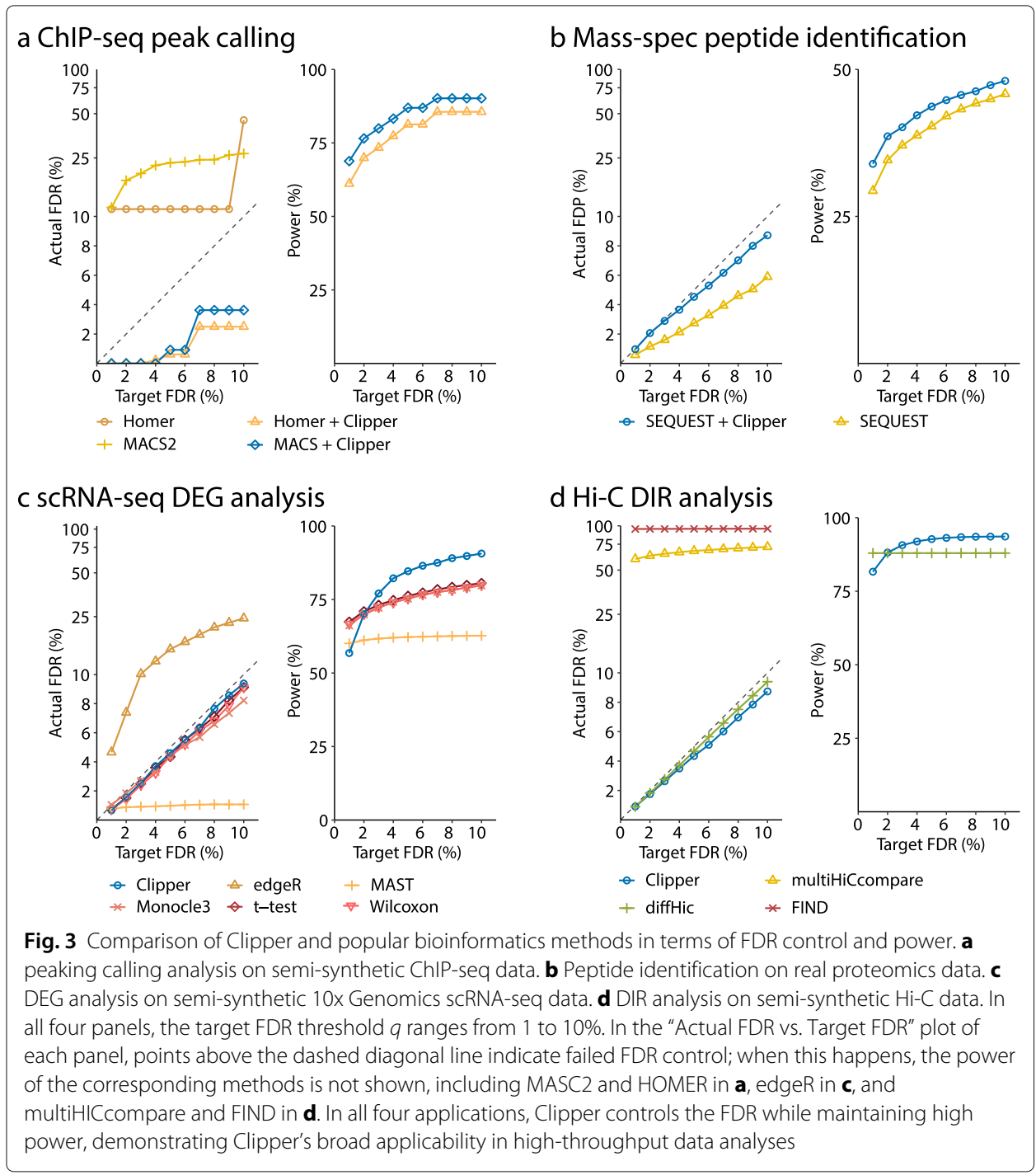


MACS2 and HOMER assume the Poisson distribution for read counts in a putative peak; however, it has been widely acknowledged that read counts are over-dispersed and thus better modeled by the negative binomial distribution [46]. Besides, MACS2 uses onesample tests to compute $p$-values when two-sample tests should be performed. As a result, the $p$-values of MACS2 and HOMER are questionable, so using their $p$-values for FDR control has no guaranteed success. (Note that MACS2 does not use $p$-values to control the FDR but instead swaps experimental and background samples to calculate the empirical FDR; yet, we emphasize that controlling the empirical FDR does not guarantee the FDR control.) As a remedy, Clipper strengthens both methods to control the FDR while maintaining high power.

As a side note, it is known that uninteresting regions tend to have larger read counts in the control sample than in the experimental (ChIP) sample; however, this phenomenon does not violate Clipper's theoretical assumption for FDR control (Additional File 1: Section S7.1).

\section{Peptide identification from MS data (enrichment analysis II)}

The state-of-the-art proteomics studies use MS experiments and database search algorithms to identify and quantify proteins in biological samples. In a typical proteomics experiment, a protein mixture sample is first digested into peptides and then measured by tandem MS technology as mass spectra, which encode peptide sequence information. "Peptide identification" is the process that decodes mass spectra and converts mass spectra into peptide sequences in a protein sequence database via search algorithms. The search process matches each mass spectrum to peptide sequences in the database and outputs the best match, called a "peptide-spectrum match" (PSM). The identified PSMs are used to infer and quantify proteins in a high-throughput manner.

False PSMs could occur when mass spectra are matched to wrong peptide sequences due to issues such as low-quality spectra, data-processing errors, and incomplete protein databases, causing problems in the downstream protein identification and quantification [47]. Therefore, a common goal of database search algorithms is to simultaneously control the FDR and maximize the number of identified PSMs, so as to maximize the number of proteins identified in a proteomics study [3, 48, 49]. A widely used FDR control strategy is the target-decoy search, where mass spectra of interest are matched to peptide sequences in both the original (target) database and a decoy database that contains artificial false protein sequences. The resulting PSMs are called the target PSMs and decoy PSMs, respectively. The decoy PSMs, i.e., matched mass spectrum and decoy peptide pairs, are known to be false and thus used by database search algorithms to control the FDR. Mainstream database search algorithms output a $q$-value for each target or decoy PSM. Discoveries are the target PSMs whose $q$-values are no greater than the target FDR threshold $q$.

We generated the first comprehensive benchmark dataset from an archaea species Pyrococcus furiosus, and we used it to examine the FDR control and power of a popular database search algorithm SEQUEST [3] (Additional File 1: Section S5.2). Using this benchmark dataset (Additional File 1: Section S6.2), we demonstrate that, as an add-on, Clipper improves the power of SEQUEST. Specifically, Clipper treats mass spectra as features. For each mass spectrum, Clipper considers the experimental (or background) measurement as the $-\log _{10}$-transformed SEQUEST $q$-value of the target (or decoy) PSM 
that includes the mass spectrum. Then Clipper decides which mass spectra and their corresponding target PSMs are discoveries (Additional File 1: Section S7.2). Based on the benchmark dataset, we examined the empirical FDR, i.e., the FDP calculated based on the true positives and negatives, and the power of SEQUEST with or without Clipper as an add-on, for a range of target FDR thresholds: $q=1 \%, 2 \%, \ldots, 10 \%$. Figure $3 \mathrm{~b}$ shows that although SEQUEST and SEQUEST+Clipper both control the FDR, SEQUEST+Clipper consistently improves the power, thus enhancing the peptide identification efficiency of proteomics experiments.

While preparing this manuscript, we found a recent work [38] that used a similar idea to identify PSMs without using $p$-values. Clipper differs from this work in two aspects: (1) Clipper is directly applicable as an add-on to any existing database search algorithms that output $q$-values and (2) Clipper is not restricted to the peptide identification application.

\section{DEG identification from bulk RNA-seq data (differential analysis I)}

RNA-seq data measure genome-wide gene expression levels in biological samples. An important use of RNA-seq data is the DEG analysis, which aims to discover genes whose expression levels change between two conditions. The FDR is a widely used criterion in DEG analysis [4-9].

We compared Clipper with two popular DEG identification methods: edgeR [4] and DESeq2 [5] (Additional File 1: Section S5.3). Specifically, when we implemented Clipper, we first performed the trimmed mean of $\mathrm{M}$ values (TMM) normalization [50] to correct for batch effects; then, we treated genes as features and their normalized expression levels as measurements under two conditions (Additional File 1: Section S7.3). We also implemented two versions of DESeq2 and edgeR: with or without IHW, a popular procedure for boosting the power of $p$-value-based FDR control methods by incorporating feature covariates [27]. In our implementation of the two versions of DESeq2 and edgeR, we used their standard pipelines, including normalization, model fitting, and gene filtering (edgeR only). To verify the FDR control, we generated four realistic semi-synthetic datasets from two real RNA-seq datasets-one from classical and non-classical human monocytes [51] and the other from yeasts with or without snf2 knockout [52] — using simulation strategies 1 and 2 (Additional File 1: Section S6.3).

In detail, in simulation strategy 1 , we used bulk RNA-seq samples from two conditions to compute a fold change for every gene between the two conditions; then, we defined true DEGs as the genes whose fold changes exceeded a threshold; next, we randomly drew three RNA-seq samples and treated them as replicates from each condition $(m=n=3$ as in the "Methods" section); using those subsampled replicates of two conditions, we preserved the true DEGs' read counts and permuted the read counts of the true nonDEGs, i.e., the genes other than true DEGs, between conditions. In summary, simulation strategy 1 guarantees that the measurements of true non-DEGs are i.i.d., an assumption that Clipper relies on for theoretical FDR control.

In simulation strategy 2, which we borrowed from a benchmark study [53], we first randomly selected at most 30\% genes as true DEGs; next, we randomly drew six RNA-seq samples from one condition (classical human monocytes and yeasts without knockout) and split the samples into two "synthetic conditions," each with three replicates ( $m=n=$ 3 as in the "Methods" section); then for each true DEG, we multiplied its read counts under one of the two synthetic conditions (randomly and independently picked for each 
gene) by a randomly generated fold change (see Additional File 1: Section S6.3); finally, for the true non-DEGs, we preserved their read counts in the six samples. In summary, simulation strategy 2 preserves batch effects, if existent in real data, for the true non-DEGs (the majority of genes). As a result, the semi-synthetic data generated under strategy 2 may violate the Clipper assumption for theoretical FDR control and thus can help evaluate the robustness of Clipper on real data.

The four semi-synthetic datasets have ground truths (true DEGs and non-DEGs) to evaluate each DEG identification method's FDR and power for a range of target FDR thresholds: $q=1 \%, 2 \%, \ldots, 10 \%$. Our results in Fig. $4 \mathrm{a}$ and Additional File 1 : Figures S15a-S17a show that Clipper consistently controls the FDR and achieves high power on all four semi-synthetic datasets. In contrast, DESeq2 and edgeR cannot consistently control the FDR except for the yeast semi-synthetic dataset generated under simulation strategy 2 . Given the fact that DESeq2 and edgeR do not consistently perform well on the three other semi-synthetic datasets, we hypothesize that their parametric distributional assumptions, if violated on real data, hinder valid FDR control (see our other study [54]), in line with our motivation for developing Clipper. By examining whether true non-DEGs' $p$-values calculated by DESeq2 or edgeR follow the theoretical Uniform $[0,1]$ distribution, we find that the answer is no for many non-DEGs, as indicated by the small $p$-values (one per non-DEG) of uniformity tests (Additional File 1: Figure S18); this issue is more serious for DESeq2, consistent with the worse FDR control of DESeq2 (Fig. 4a and Additional File 1: Figures S15a-S17a). Furthermore, we observe that adding IHW to edgeR and DESeq2 has negligible effects on the four semi-synthetic datasets.

To further explain why DESeq2 fails to control the FDR, we examined the $p$-value distributions of 16 non-DEGs that were most frequently identified (from the $100 \mathrm{semi}$ synthetic datasets generated from the human monocyte dataset using simulation strategy 1) by DESeq2 at the target FDR threshold $q=0.05$. Our results in Additional File 1 : Figure S19 show that the 16 non-DEGs' $p$-values are non-uniformly distributed with a mode close to 0 . Such unusual enrichment of overly small $p$-values makes these non-DEGs mistakenly called as discoveries by DESeq2.

In addition, we compared the DEG ranking by Clipper, edgeR, and DESeq2 in two ways. First, for true DEGs, we compared their ranking by each method with their true ranking based on true expression fold changes (from large to small, as in semi-synthetic data generation in Additional File 1: Section S6.3). Specifically, we ranked true DEGs using Clipper's contrast scores (from large to small), edgeR's $p$-values (from small to large), or DESeq2's $p$-values (from small to large). Our results in Fig. 4b and Additional File 1: Figures S15b-S17b show that Clipper's contrast scores exhibit the most consistent ranking with the ranking based on true fold changes. Second, to compare the power of Clipper, edgeR, and DESeq2 based on their DEG rankings instead of nominal $p$-values, we calculated their power under the actual FDRs, which only depend on gene rankings (for the definition of actual FDR, see Additional File 1: Section S6.3). Figure 4a and Additional File 1: Figures S15a-S17a show that, when Clipper, edgeR, and DESeq2 have the same actual FDR, Clipper consistently outperforms edgeR and DESeq2 in terms of power, i.e., Clipper has the largest number of true DEGs in its top ranked genes.

We also compared the reproducibility of Clipper, edgeR, and DESeq2 in the presence of sampling randomness. Specifically, we used two semi-synthetic datasets (generated independently from the same procedure in Additional File 1: Section S6.3) as technical 
Human monocyte semi-synthetic datasets by simulation strategy 2

a

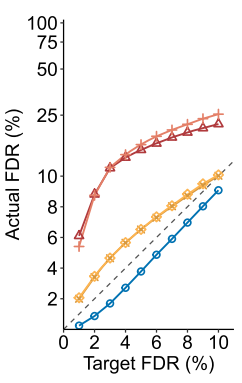

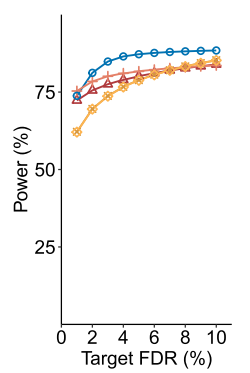

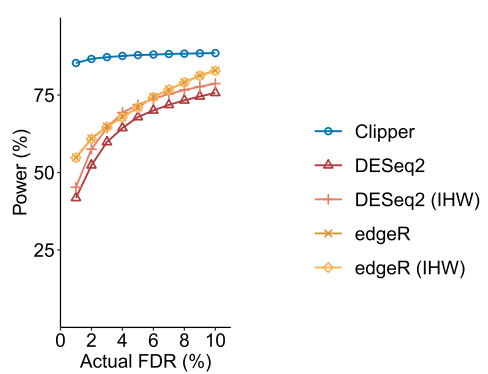

b

True DEGs among top 100 DEGs identified by each DE method
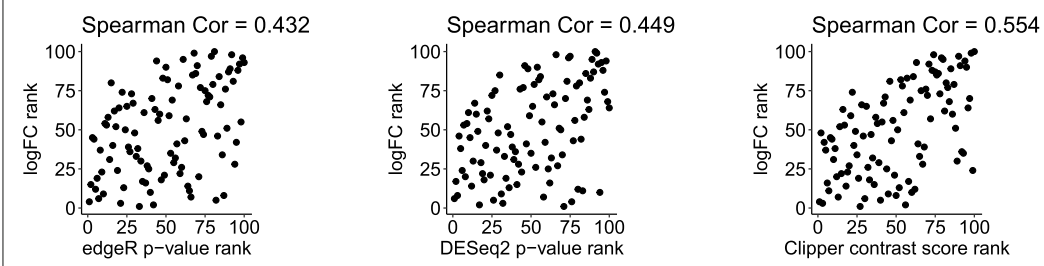

C True DEGs identified among the top 100 DEGs in dataset 1 or dataset 2
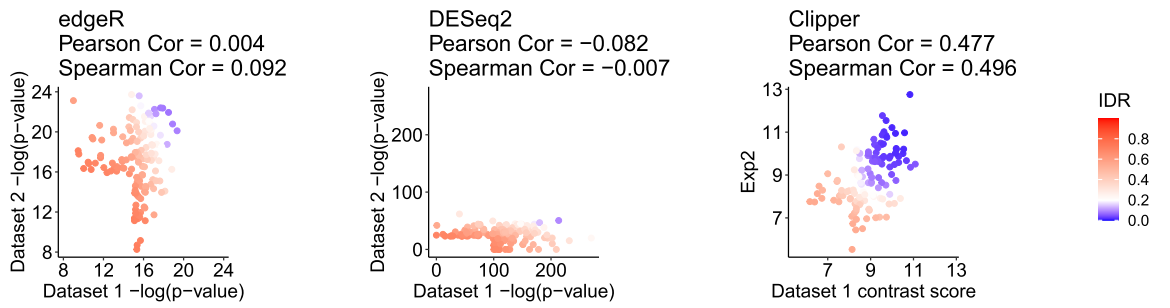

Fig. 4 Comparison of Clipper and two popular DEG identification methods_edgeR and DESeq2—in DEG analysis on semi-synthetic bulk RNA-seq data (generated from human monocyte real data using simulation strategy 2 in Additional File 1: Section S6.3). a FDR control, power given the same target FDR, and power given the same actual FDR. b Ranking consistency of the true DEGs among the top 100 DEGs identified by each method. The consistency is defined between the genes' ranking based on edgeR/DESeq2's $p$-values or Clipper's contrast scores and their ranking based on true expression fold changes. c Reproducibility between two semi-synthetic datasets as technical replicates. Three reproducibility criteria are used: the IDR, the Pearson correlation, and the Spearman correlation. Each criterion is calculated for edgeR/DESeq2's p-values or Clipper's contrast scores on the two semi-synthetic datasets. Among the three methods, only Clipper controls the FDR, and Clipper achieves the highest power, the best gene ranking consistency, and the best reproducibility

replicates and computed Clipper's contrast scores, edgeR's $p$-values, and DESeq's $p$-values on each dataset. For each method, we evaluated its reproducibility between the two semisynthetic datasets by computing three criteria-the irreproducibility discovery rate (IDR) [55], the Pearson correlation, and the Spearman correlation-using its contrast scores or $-\log _{10}$-transformed $p$-values. Figure $4 \mathrm{c}$ and Additional File 1: Figures S15c-S17c show that Clipper's contrast scores have higher reproducibility by all three criteria compared to edgeR's and DESeq2's $p$-values.

Finally, we compared Clipper with DESeq2 and edgeR on the real RNA-seq data of classical and non-classical human monocytes [51]. In this dataset, gene expression changes 
are expected to be associated with the immune response process. We input three classical and three non-classical samples into Clipper, DESeq2, and edgeR for DEG identification. Figure 5a shows that edgeR identifies the fewest DEGs, while DESeq2 identifies the most DEGs, followed by Clipper. Notably, most DEGs identified by DESeq2 are not identified by Clipper or edgeR. To investigate whether DESeq2 makes too many false discoveries and whether Clipper finds biologically meaningful DEGs missed by DESeq2 or edgeR, we performed functional analysis on the set of DEGs identified by each method. We first performed the gene ontology (GO) analysis on the three sets of identified DEGs using the R package clusterProfiler [56]. Figure 5b ("Total") shows that more GO terms are enriched (with enrichment q-values $\leq 0.01$ ) in the DEGs identified by Clipper than in the DEGs identified by DESeq2 or edgeR. For the GO terms enriched in all three sets of identified DEGs, Fig. $5 \mathrm{c}$ shows that they are all related to the immune response and thus biologically meaningful. Notably, these biologically meaningful GO terms have more significant enrichment in Clipper's identified DEGs than in edgeR and DESeq2's identified DEGs. We further performed GO analysis on the DEGs uniquely identified by one method in pairwise comparisons of Clipper vs. DESeq2 and Clipper vs. edgeR. Figure $5 \mathrm{~b}$ and Additional File 1: Figure $\mathrm{S} 20$ show that multiple immune-related GO terms are enriched in Clipper-specific DEGs, while no GO terms are enriched in edgeR-specific or DESeq2-specific DEGs. In addition, we examined the DEGs that were identified by Clipper only but missed by both edgeR and DESeq2. Figure $5 \mathrm{~d}$ and Additional File 2 show that these genes include multiple key immune-related genes, including CD36, DUSP2, and TNFAIP3. We further performed pathway analysis on these genes and the DEGs that were identified by DEseq2 only but missed by both edgeR and Clipper, using the $\mathrm{R}$ package limma [10]. Additional File 1: Figure S21a shows that the DEGs that were only identified by Clipper have significant enrichment for immune-related pathways including phagosome, a key function of monocytes and macrophages. On the contrary, Additional File 1: Figure S21b shows that fewer immune-related pathways are enriched in DEGs that were only identified by DESeq2. Altogether, these results confirm the capacity of Clipper in real-data DEG analysis, and they are consistent with our simulation results that edgeR lacks power, while DESeq2 fails to control the FDR.

\section{DEG identification from single-cell RNA-seq data (differential analysis II)}

Single-cell RNA sequencing (scRNA-seq) technologies have revolutionized biomedical sciences by enabling genome-wide profiling of gene expression levels at an unprecedented single-cell resolution. DEG analysis is widely applied to scRNA-seq data for discovering genes whose expression levels change between two conditions or between two cell types. Compared with bulk RNA-seq data, scRNA-seq data have many more "replicates" (i.e., cells, whose number is often in hundreds) under each condition or within each cell type.

We compared Clipper (Additional File 1: Section S7.4) with edgeR [4], MAST [57], Monocle3 [58], the two-sample $t$ test, and the Wilcoxon rank-sum test (Additional File 1: Section S5.4), five methods that are either popular or reported to have comparatively top performance from a previous benchmark study [59]. To verify the FDR control, we used scDesign2, a flexible probabilistic simulator, to generate scRNA-seq count data with known true DEGs [60]. scDesign2 offers three key advantages that enable the generation of realistic semi-synthetic scRNA-seq count data: (1) it captures distinct marginal distributions of different genes, (2) it preserves gene-gene correlations, and (3) it adapts to 


\section{a}

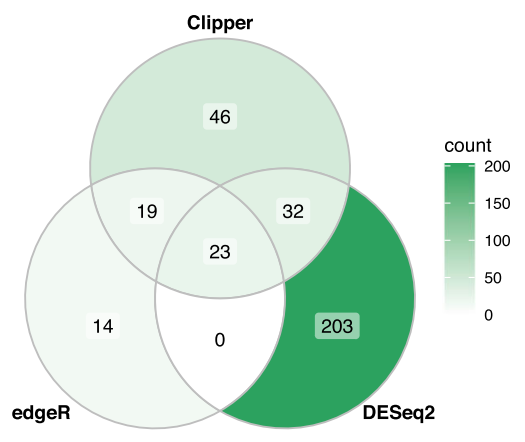

b

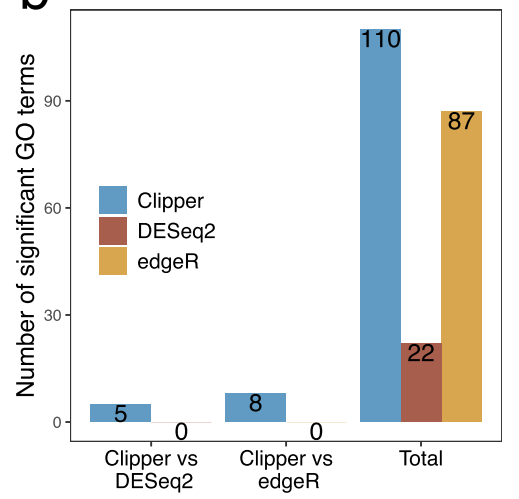

C

Top GO terms enriched in all three sets of identified DEGs

\begin{tabular}{|c|c|c|c|}
\hline GO term (ID) & qvalue (DESeq2) & qvalue (edgeR) & qvalue (Clipper) \\
\hline leukocyte chemotaxis (GO:0030595) & $9.930044 \mathrm{e}-06$ & $9.594885 \mathrm{e}-09$ & $3.104557 \mathrm{e}-10$ \\
\hline myeloid leukocyte migration (GO:0097529) & $1.107612 \mathrm{e}-05$ & $2.921486 \mathrm{e}-08$ & $5.740217 \mathrm{e}-10$ \\
\hline granulocyte chemotaxis (GO:0071621) & $2.698853 \mathrm{e}-05$ & $1.008808 \mathrm{e}-08$ & $1.167108 \mathrm{e}-09$ \\
\hline neutrophil chemotaxis (GO:0030593) & $2.698853 \mathrm{e}-05$ & $2.921486 \mathrm{e}-08$ & $2.691033 \mathrm{e}-09$ \\
\hline
\end{tabular}

d

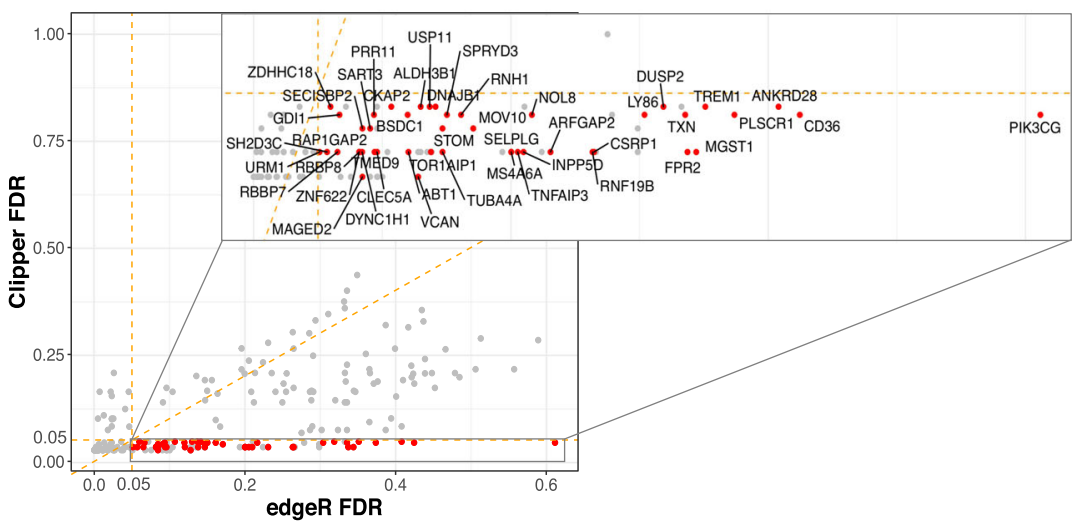

Fig. 5 Application of Clipper, DESeq2, and edgeR to identifying DEGs from the classical and non-classical human monocyte dataset. a A Venn diagram showing the overlaps of the identified DEGs (at the FDR threshold $q=5 \%$ ) by the three DE methods. b Numbers of GO terms enriched (with enrichment $q$-values $<0.01$ ) in the DEGs found by Clipper, DESeq2, and edgeR (column 3), or in the DEGs specifically identified by Clipper or DESeq2/edgeR in the pairwise comparison between Clipper and DESeq2 (column 1) or between Clipper and edgeR (column 2). More GO terms are enriched in the DEGs identified by Clipper than in those identified by edgeR or DESeq2. c Enrichment q-values of four GO terms that are found enriched (with enrichment $q$-values $<0.01$ ) in all three sets of identified DEGs, one set per method. All the four terms are most enriched in the DEGs identified by Clipper. $\mathbf{d}$ A scatterplot of the claimed FDR of Clipper against that of edgeR for all the DEGs identified by Clipper, edgeR or DESeq2. The 46 DEGs identified by Clipper only at 5\% FDR are highlighted in red

various scRNA-seq protocols. Using scDesign2, we generated two semi-synthetic scRNAseq datasets from two real scRNA-seq datasets of peripheral blood mononuclear cells (PBMCs) [61]: one using 10x Genomics [62] and the other using Drop-seq [63]. Each semi-synthetic dataset contains two cell types, CD4+ T cells and cytotoxic T cells, which we treated as two conditions (Additional File 1: Section S6.4). Having true DEGs known, 
the semi-synthetic datasets allow us to evaluate the FDRs and power of Clipper and the other five methods for a range of target FDR thresholds: $q=1 \%, 2 \%, \ldots, 10 \%$. Figure 3c and Additional File 1: Figure S22 show that on both 10x Genomics and Drop-seq semisynthetic datasets, Clipper consistently controls the FDR and remains the most powerful (except for $q=1 \%$ and $2 \%$ ) among all the methods that achieve FDR control. These results demonstrate Clipper's robust performance in scRNA-seq DEG analysis.

\section{DIR analysis of Hi-C data (differential analysis III)}

$\mathrm{Hi}-\mathrm{C}$ experiments are widely used to investigate spatial organizations of chromosomes and to map chromatin interactions across the genome. A Hi-C dataset is often processed and summarized into an interaction matrix, whose rows and columns represent manually binned chromosomal regions and whose $(i, j)$-th entry represents the measured contact intensity between the $i$-th and $j$-th binned regions. The DIR analysis aims to identify pairs of genomic regions whose contact intensities differ between conditions. Same as DEG analysis, DIR analysis also uses the FDR as a decision criterion [11-13].

We compared Clipper with three popular DIR identification methods: diffHic [13], FIND [12], and multiHiCcompare [11] (Additional File 1: Section S5.5). Specifically, we applied Clipper to DIR identification by treating pairs of genomic regions as features and their contact intensities as measurements. To verify the FDR control of Clipper (Additional File 1: Section S7.5), diffHic, FIND, and multiHiCcompare, we generated realistic semi-synthetic data from real interaction matrices of ENCODE cell line GM12878 [44] with true spiked-in DIRs to evaluate the FDR and power (Additional File 1: Section S6.5). We examined the actual FDR and power in a range of target FDR thresholds: $q=$ $1 \%, 2 \%, \ldots, 10 \%$. Figure $3 \mathrm{~d}$ shows that Clipper and diffHic are the only two methods that consistently control the FDR, while multiHiCcompare and FIND fail by a large margin. In terms of power, Clipper outperforms diffHic except for $q=1 \%$ and $2 \%$, even though Clipper has not been optimized for $\mathrm{Hi}-\mathrm{C}$ data analysis. This result demonstrates Clipper's general applicability and strong potential for DIR analysis.

\section{Discussion}

In this paper, we proposed a new statistical framework, Clipper, for identifying interesting features with FDR control from high-throughput data. Clipper avoids the use of $p$-values and makes FDR control more reliable and flexible. We used comprehensive simulation studies to verify the FDR control by Clipper under various settings. We demonstrate that Clipper outperforms existing generic FDR control methods by having higher power and greater robustness to model misspecification. We further applied Clipper to four popular bioinformatics analyses: peak calling from ChIP-seq data, peptide identification from MS data, DEG identification from RNA-seq data, and DIR identification from Hi-C data. Our results indicate that Clipper provides a powerful add-on to existing bioinformatics tools to improve the reliability of FDR control and thus the reproducibility of scientific discoveries.

Clipper's FDR control procedures (BC and GZ procedures in the "Methods" section) are motivated by the Barber-Candès (BC)'s knockoff paper [35] and the Gimenez-Zou (GZ)'s multiple knockoff paper [64], but we do not need to construct knockoffs in enrichment analysis when two conditions have the same number of replicates; the reason is that the replicates under the background condition serve as natural negative 
controls. For differential analysis and enrichment analysis with unequal numbers of replicates, in order to guarantee the theoretical assumptions for FDR control, Clipper uses permutations instead of the complicated knockoff construction because Clipper only examines features marginally and does not concern about features' joint distribution.

We validated the FDR control by Clipper using extensive and concrete simulations, including both model-based and real-data-based data generation with ground truths, which are widely used to validate newly developed computational frameworks [65]. In contrast, in most bioinformatics method papers, the FDR control was merely mentioned but rarely validated. Many of them assumed that using the $\mathrm{BH}$ procedure on $p$-values would lead to valid FDR control; however, the reality is often otherwise because $p$-values would be invalid when model assumptions were violated or the $p$-value calculation was problematic. Here we voice the importance of validating FDR control in bioinformatics method development, and we use this work as a demonstration. We believe that Clipper provides a powerful booster to this movement. As a $p$-value-free alternative to the classic $p$-value-based $\mathrm{BH}$ procedure, Clipper relies less on model assumptions and is thus more robust to model misspecifications, making it an appealing choice for FDR control in diverse high-throughput biomedical data analyses.

Clipper is a flexible framework that is easily generalizable to identify a variety of interesting features. The core component of Clipper summarizes each feature's measurements under each condition into an informative statistic (e.g., the sample mean); then Clipper combines each feature's informative statistics under two conditions into a contrast score to enable FDR control. The current implementation of Clipper only uses the sample mean as the informative statistic to identify the interesting features that have distinct expected values under two conditions. However, by modifying the informative statistic, we can generalize Clipper to identify the features that are interesting in other aspects, e.g., having different variances between two conditions. Regarding the contrast score, Clipper currently makes careful choices between two contrast scores, minus and maximum, based on the number of replicates and the analysis task (enrichment or differential).

Notably, Clipper achieves FDR control and high power using those two simple contrast scores, which are calculated for individual features without borrowing information from other features. However, Clipper does leverage the power of multiple testing by setting a contrast score threshold based on all features' contrast scores. This is a likely reason why Clipper achieves good power even with simple contrast scores. An advantage of Clipper is that it allows other definitions of contrast scores, such as the two-sample $t$ statistic that considers within-condition variances. Empirical evidence (Additional File 1: Figures S13 and S14) shows that the Clipper variant using the two-sample $t$ statistic is underpowered by the default Clipper, which uses the minus summary statistic (difference of two conditions' sample means) as the contrast score in the 3vs3 enrichment analysis or as the degree of interestingness in the 3vs3 differential analysis (see the "Methods" section). Here is our current interpretation of this seemingly counter-intuitive result.

- First, both the minus statistic and the $t$ statistic satisfy Clipper's theoretical conditions (Lemmas 1 and 3 in Additional File 1: Section S2), which guarantee the FDR control by the $\mathrm{BC}$ and GZ procedures; this is confirmed in Additional File 1: Figures S13 and S14. Hence, from the FDR control perspective, Clipper does not require the adjustment for within-condition variances by using a $t$ statistic. 
- Second, Clipper is different from the two-sample $t$ test or the regression-based $t$ test, where the $t$ statistic was purposely derived as a pivotal statistic so that its null distribution (the $t$ distribution) does not depend on unknown parameters. Since Clipper does not require a null distribution for each feature, the advantage of the $t$ statistic being pivotal no longer matters.

- Third, the minus statistic only requires estimates of two conditions' mean parameters, while the $t$ statistic additionally requires estimates of the two conditions' variances. Hence, when the sample sizes (i.e., the numbers of replicates) are small, the two more parameters that need estimation in the $t$ statistic might contribute to the observed power loss of the Clipper $t$ statistic variant. Indeed, the power difference between the two statistics diminishes as the sample sizes increase from 3vs3 in Additional File 1: Figures S13-S14 to 10vs10 in Additional File 1: Figure S10 (where we compared the default Clipper with BH-pair-parametric, which is based on the two-sample $t$ test and is highly similar to the Clipper $t$ statistic variant).

- Fourth, we observe empirically that a contrast score would have better power if its distribution (based on its values of all features) has a larger range and a heavier right tail (in the positive domain). Compared to the minus statistic, the $t$ statistic has a smaller range and a lighter right tail due to its adjustment for features' within-condition variances (Additional File 1: Figure S23). This observation is consistent with the power difference of the two statistics.

Beyond our current interpretation, however, we admit that future studies are needed to explore alternative contrast scores and their power with respect to data characteristics and analysis tasks. Furthermore, we may generalize Clipper to be robust against sample batch effects by constructing the contrast score as a regression-based test statistic that has batch effects removed.

Our current version of Clipper allows the identification of interesting features between two conditions. However, there is a growing need to generalize our framework to identify features across more than two conditions. For example, temporal analysis of scRNA-seq data aims to identify genes whose expression levels change along cell pseudotime [31]. To tailor Clipper for such analysis, we could define a new contrast score that differentiates the genes with stationary expression (uninteresting features) from the other genes with varying expression (interesting features). Further studies are needed to explore the possibility of extending Clipper to the regression framework so that Clipper can accommodate data of multiple conditions or even continuous conditions, as well as adjusting for confounding covariates.

We have demonstrated the broad application potential of Clipper in various bioinformatics data analyses. Specifically, when used as an add-on to established, popular bioinformatics methods such as MACS2 for peak calling and SEQUEST for peptide identification, Clipper guaranteed the desired FDR control and in some cases boosted the power. However, many more careful thoughts are needed to escalate Clipper into standalone bioinformatics methods for specific data analyses, for which data processing and characteristics (e.g., peak lengths, GC contents, proportions of zeros, and batch effects) must be appropriately accounted for before Clipper is used for the FDR control $[59,66]$. We expect that the Clipper framework will propel future development of bioinformatics 
methods by providing a flexible $p$-value-free approach to control the FDR, thus improving the reliability of scientific discoveries.

After finishing this manuscript, we were informed of the work by He et al. [67], which is highly similar to the part of Clipper for differential analysis, as both work use permutation for generating negative controls and the GZ procedure for thresholding (test statistics in $\mathrm{He}$ et al. and contrast scores in Clipper). However, the test statistics used in $\mathrm{He}$ et al. are the two-sample $t$ statistic and the two-sample Wilcoxon statistic, both of which are different from the minus and maximum contrast scores used in Clipper. While we leave the optimization of contrast scores to future work, we observed that the minus contrast score outpowers the two-sample $t$ statistic in our analysis (Additional File 1: Figures S13 and S14), and we hypothesize that the two-sample Wilcoxon statistic, though being a valid contrast score for differential analysis, requires a large sample size to achieve good power. For this reason, we did not consider it as a contrast score in the current Clipper implementation, whose focus is on small-sample-size high-throughout biological data.

\section{Conclusion}

In high-throughput biological data analysis, which aims to identify interesting features by comparing two conditions, existing bioinformatics tools control the FDR based on $p$ values. However, obtaining valid $p$-values relies on either reasonable assumptions of data distribution or large numbers of replicates under both conditions-two requirements that are often unmet in biological studies. To address this issue, we propose Clipper, a general statistical framework for FDR control without relying on $p$-values or specific data distributions. Clipper is applicable to identifying both enriched and differential features from high-throughput biological data of diverse types. In comprehensive simulation and real-data benchmarking, Clipper outperforms existing generic FDR control methods and specific bioinformatics tools designed for various tasks, including peak calling from ChIP-seq data, differentially expressed gene identification from bulk or singlecell RNA-seq data, differentially interacting chromatin region identification from $\mathrm{Hi}-\mathrm{C}$ data, and peptide identification from mass spectrometry data. Our results demonstrate Clipper's flexibility and reliability for FDR control, as well as its broad applications in high-throughput data analysis.

\section{Methods}

\section{Clipper: notations and assumptions}

We first introduce notations and assumptions used in Clipper. While the differential analysis treats the two conditions symmetric, the enrichment analysis requires one condition to be the experimental condition (i.e., the condition of interest) and the other condition to be the background condition (i.e., the negative control). For simplicity, we use the same set of notations for both analyses. For two random vectors $X=\left(X_{1}, \ldots, X_{m}\right)^{\top}$ and $\boldsymbol{Y}=\left(Y_{1}, \ldots, Y_{n}\right)^{\top}$, we write $\boldsymbol{X} \perp \boldsymbol{Y}$ if $X_{i}$ is independent of $Y_{j}$ for all $i=1, \ldots, m$ and $j=1, \ldots, n$. To avoid confusion, we use card $(A)$ to denote the cardinality of a set $A$ and $|c|$ to denote the absolute value of a scalar $c$. We define $a \vee b:=\max (a, b)$.

Clipper only requires two inputs: the target FDR threshold $q \in(0,1)$ and the input data. Regarding the input data, we use $d$ to denote the number of features with measurements under two conditions, and we use $m$ and $n$ to denote the numbers of replicates under the two conditions. For each feature $j=1, \ldots, d$, we use $X_{j}=\left(X_{j 1}, \ldots, X_{j m}\right)^{\top} \in \mathbb{R}_{\geq 0}^{m}$ and 
$\boldsymbol{Y}_{j}=\left(Y_{j 1}, \ldots, Y_{j n}\right)^{\top} \in \mathbb{R}_{\geq 0}^{n}$ to denote its measurements under the two conditions, where $\mathbb{R}_{\geq 0}$ denotes the set of non-negative real numbers. We assume that all measurements are non-negative, as is the case with most high-throughput experiments. (If this assumption does not hold, transformations can be applied to make data satisfy this assumption.)

Clipper has the following assumptions on the joint distribution of $\boldsymbol{X}_{1}, \ldots, \boldsymbol{X}_{d}, \boldsymbol{Y}_{1}, \ldots, \boldsymbol{Y}_{d}$. For $j=1, \ldots, d$, Clipper assumes that $X_{j 1}, \ldots, X_{j m}$ are identically distributed, so are $Y_{j 1}, \ldots, Y_{j n}$. Let $\mu_{X j}=\mathbb{E}\left[X_{j 1}\right]$ and $\mu_{Y j}=\mathbb{E}\left[Y_{j 1}\right]$ denote the expected measurement of feature $j$ under the two conditions, respectively. Then conditioning on $\left\{\mu_{X j}\right\}_{j=1}^{d}$ and $\left\{\mu_{Y j}\right\}_{j=1}^{d}$,

$$
\begin{array}{r}
X_{j 1}, \cdots, X_{j m}, Y_{j 1}, \cdots, Y_{j n} \text { are mutually independent } ; \\
\boldsymbol{X}_{j} \perp \boldsymbol{X}_{k}, \boldsymbol{Y}_{j} \perp \boldsymbol{Y}_{k} \text { and } \boldsymbol{X}_{j} \perp \boldsymbol{Y}_{k}, \forall j, k=1, \ldots, d .
\end{array}
$$

An enrichment analysis aims to identify interesting features with $\mu_{X j}>\mu_{Y j}$ (with $X_{j}$ and $\boldsymbol{Y}_{j}$ defined as the measurements under the experimental and background conditions, respectively), while a differential analysis aims to call interesting features with $\mu_{X j} \neq \mu_{Y j}$. We define $\mathcal{N}:=\left\{j: \mu_{X j}=\mu_{Y j}\right\}$ as the set of uninteresting features and denote $N:=$ $\operatorname{card}(\mathcal{N})$. In both analyses, Clipper further assumes that an uninteresting feature $j$ satisfies

$$
X_{j 1}, \cdots, X_{j m}, Y_{j 1}, \cdots, Y_{j n} \text { are identically distributed }, \forall j \in \mathcal{N} .
$$

Clipper consists of two main steps: construction and thresholding of contrast scores. First, Clipper computes contrast scores, one per feature, as summary statistics that reflect the extent to which features are interesting. Second, Clipper establishes a contrast-score cutoff and calls as discoveries the features whose contrast scores exceed the cutoff.

To construct contrast scores, Clipper uses two summary statistics $t(\cdot, \cdot): \mathbb{R}_{\geq 0}^{m} \times \mathbb{R}_{\geq 0}^{n} \rightarrow \mathbb{R}$ to extract data information regarding whether a feature is interesting or not:

$$
\begin{aligned}
t^{\text {minus }}(\boldsymbol{x}, \boldsymbol{y}) & :=\bar{x}-\bar{y} ; \\
t^{\max }(\boldsymbol{x}, \boldsymbol{y}) & :=\max (\bar{x}, \bar{y}) \cdot \operatorname{sign}(\bar{x}-\bar{y}),
\end{aligned}
$$

where $\boldsymbol{x}=\left(x_{1}, \ldots, x_{m}\right)^{\top} \in \mathbb{R}_{\geq 0}^{m}, \boldsymbol{y}=\left(y_{1}, \ldots, y_{n}\right)^{\top} \in \mathbb{R}_{\geq 0}^{n}, \bar{x}=\sum_{i=1}^{m} x_{i} / m, \bar{y}=\sum_{i=1}^{n} y_{i} / n$, and $\operatorname{sign}(\cdot): \mathbb{R} \rightarrow\{-1,0,1\}$ with $\operatorname{sign}(x)=1$ if $x>0$, $\operatorname{sign}(x)=-1$ if $x<0$, and $\operatorname{sign}(x)=0$ otherwise.

Notably, other summary statistics can also be used to construct contrast scores. For example, an alternative summary statistic is the $t$ statistic from the two-sample $t$ test:

$$
t^{\mathrm{t}}(\boldsymbol{x}, \boldsymbol{y}):=\frac{\bar{x}-\bar{y}}{\sqrt{\frac{\sum_{i=1}^{m}\left(x_{i}-\bar{x}\right)^{2}+\sum_{i=1}^{n}\left(y_{i}-\bar{y}\right)^{2}}{m+n-2}}} .
$$

Then we introduce how Clipper works in three analysis tasks: the enrichment analysis with equal numbers of replicates under two conditions $(m=n)$, the enrichment analysis with different numbers of replicates under two conditions $(m \neq n)$, and the differential analysis (when $m+n>2$ ).

\section{Clipper: enrichment analysis with equal numbers of replicates $(m=n)$}

Under the enrichment analysis, we assume that $\boldsymbol{X}_{j} \in \mathbb{R}_{\geq 0}^{m}$ and $\boldsymbol{Y}_{j} \in \mathbb{R}_{\geq 0}^{n}$ are the measurements of feature $j, j=1, \ldots, d$, under the experimental and background conditions 
with $m$ and $n$ replicates, respectively. We start with the simple case when $m=n$. Clipper defines a contrast score $C_{j}$ of feature $j$ in one of two ways:

$$
C_{j}:=t^{\text {minus }}\left(\boldsymbol{X}_{j}, \boldsymbol{Y}_{j}\right) \quad \text { minus contrast score },
$$

or

$$
C_{j}:=t^{\max }\left(\boldsymbol{X}_{j}, Y_{j}\right) \quad \text { maximum contrast score } .
$$

Figure 6a shows a cartoon illustration of contrast scores when $m=n=1$. Accordingly, a large positive value of $C_{j}$ bears evidence that $\mu_{X j}>\mu_{Y j}$. Motivated by [35], Clipper uses the following procedure to control the FDR under the target level $q \in(0,1)$.

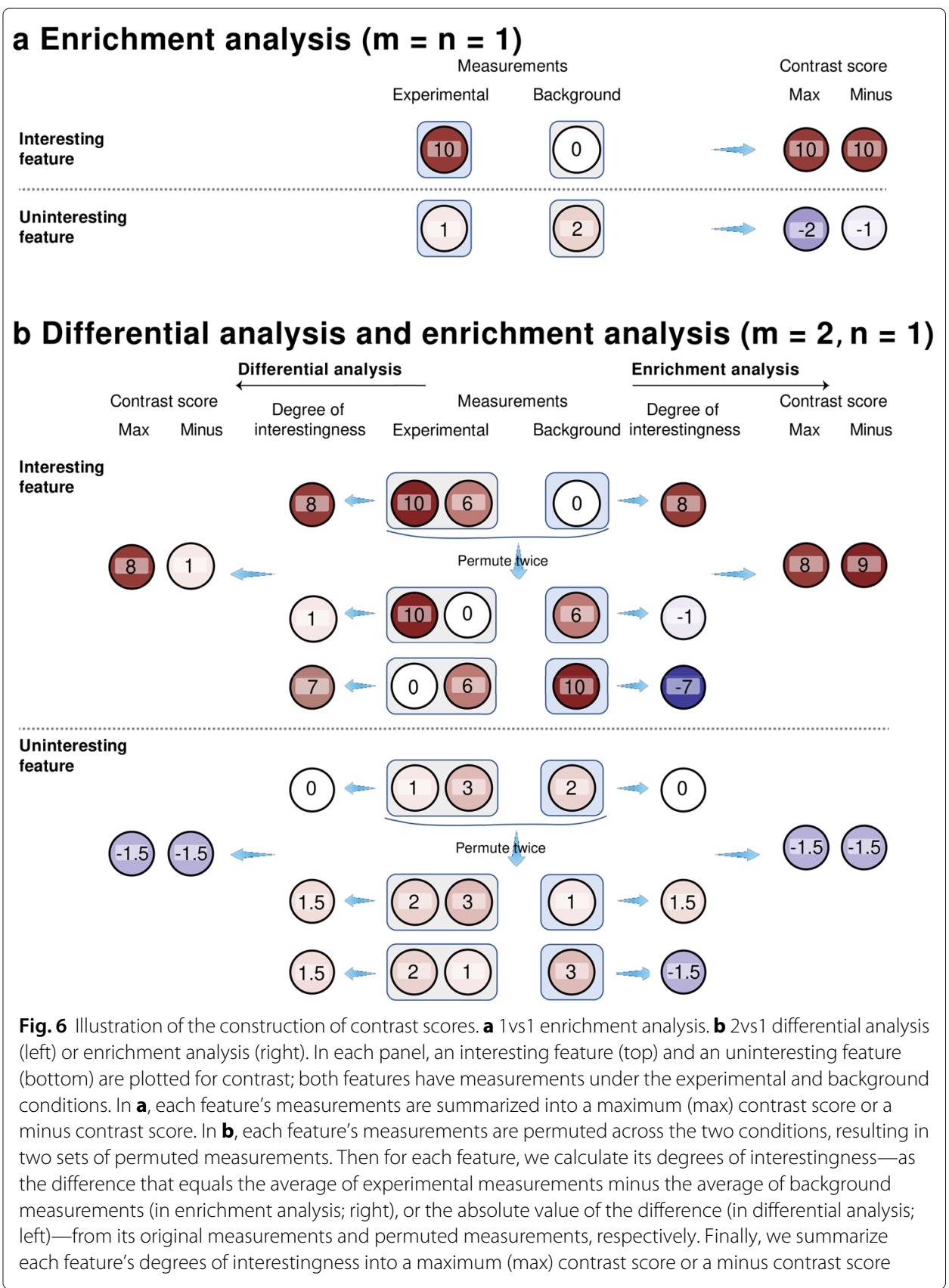


Definition 1 (Barber-Candès $(\mathrm{BC})$ procedure for thresholding contrast scores [35]) Given contrast scores $\left\{C_{j}\right\}_{j=1}^{d}, \mathcal{C}=\left\{\left|C_{j}\right|: C_{j} \neq 0 ; j=1, \ldots, d\right\}$ is defined as the set of non-zero absolute values of $C_{j}$ 's. The $B C$ procedure finds a contrast-score cutoff $T^{B C}$ based on the target FDR threshold $q \in(0,1)$ as

$$
T^{B C}:=\min \left\{t \in \mathcal{C}: \frac{\operatorname{card}\left(\left\{j: C_{j} \leq-t\right\}\right)+1}{\operatorname{card}\left(\left\{j: C_{j} \geq t\right\}\right) \vee 1} \leq q\right\}
$$

and outputs $\left\{j: C_{j} \geq T^{B C}\right\}$ as discoveries.

\section{Clipper: enrichment analysis with any numbers of replicates $\boldsymbol{m}$ and $\boldsymbol{n}$}

When $m \neq n$, Clipper constructs contrast scores via permutation of replicates across conditions. The idea is that, after permutation, every feature becomes uninteresting and can serve as its own negative control.

Definition 2 (Permutation) We define $\sigma$ as permutation, i.e., a bijection from the set $\{1, \cdots, m+n\}$ onto itself, and we rewrite the data $X_{1}, \ldots, X_{d}, Y_{1}, \ldots, Y_{d}$ into a matrix $\mathbf{W}$ :

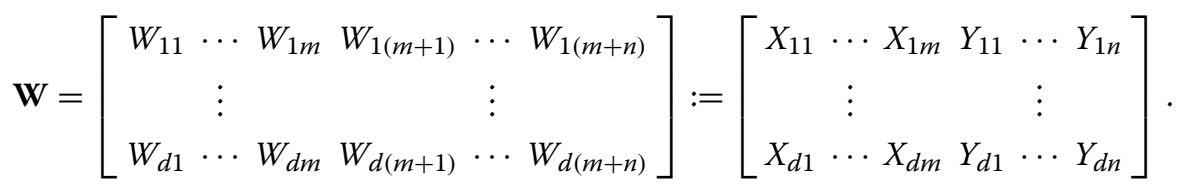

We then apply $\sigma$ to permute the columns of $\mathbf{W}$ and obtain

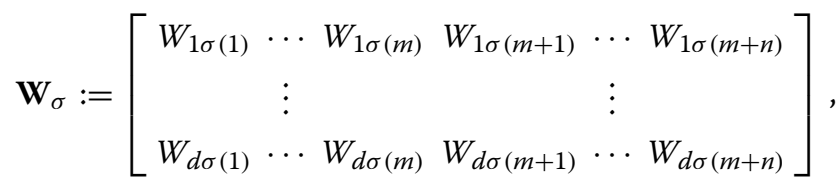

from which we obtain the permuted measurements $\left\{\left(X_{j}^{\sigma}, Y_{j}^{\sigma}\right)\right\}_{j=1}^{d}$, where

$$
\begin{aligned}
X_{j}^{\sigma} & :=\left(W_{j \sigma(1)}, \ldots, W_{j \sigma(m)}\right)^{\top}, \\
\boldsymbol{Y}_{j}^{\sigma} & :=\left(W_{j \sigma(m+1)}, \ldots, W_{j \sigma(m+n)}\right)^{\top} .
\end{aligned}
$$

In the enrichment analysis, if two permutations $\sigma$ and $\sigma^{\prime}$ satisfy that

$$
\{\sigma(1), \cdots, \sigma(m)\}=\left\{\sigma^{\prime}(1), \cdots, \sigma^{\prime}(m)\right\},
$$

then we define $\sigma$ and $\sigma^{\prime}$ to be in one equivalence class. That is, permutations in the same equivalence class lead to the same division of $m+n$ replicates (from the two conditions) into two groups with sizes $m$ and $n$. In total, there are $\left(\begin{array}{c}m+n \\ m\end{array}\right)$ equivalence classes of permutations.

We define $\sigma_{0}$ as the identity permutation such that $\sigma_{0}(i)=i$ for all $i \in\{1, \cdots, m+n\}$. In addition, Clipper randomly samples $h$ equivalence classes $\sigma_{1}, \ldots, \sigma_{h}$ with equal probabilities without replacement from the other $h_{\max }:=\left(\begin{array}{c}m+n \\ m\end{array}\right)-1$ equivalence classes (after excluding the equivalence class containing $\sigma_{0}$ ). Note that $h_{\max }$ is the maximum value $h$ can take.

Clipper then obtains $\left\{\left(\boldsymbol{X}_{j}^{\sigma_{0}}, \boldsymbol{Y}_{j}^{\sigma_{0}}\right),\left(\boldsymbol{X}_{j}^{\sigma_{1}}, \boldsymbol{Y}_{j}^{\sigma_{1}}\right), \ldots,\left(\boldsymbol{X}_{j}^{\sigma_{h}}, \boldsymbol{Y}_{j}^{\sigma_{h}}\right)\right\}_{j=1}^{d}$, where $\left(\boldsymbol{X}_{j}^{\sigma_{\ell}}, \boldsymbol{Y}_{j}^{\sigma_{\ell}}\right)$ are the permuted measurements based on $\sigma_{\ell}, \ell=0,1, \ldots, h$. Then Clipper computes $T_{j}^{\sigma_{\ell}}:=t^{\text {minus }}\left(\boldsymbol{X}_{j}^{\sigma_{\ell}}, \boldsymbol{Y}_{j}^{\sigma_{\ell}}\right)$ to indicate the degree of "interestingness" of feature 
$j$ reflected by $\left(\boldsymbol{X}_{j}^{\sigma_{\ell}}, \boldsymbol{Y}_{j}^{\sigma_{\ell}}\right)$. Note that Clipper chooses $t^{\text {minus }}$ instead of $t^{\max }$ because empirical evidence shows that $t^{\text {minus }}$ leads to better power. Sorting $\left\{T_{j}^{\sigma_{\ell}}\right\}_{\ell=0}^{h}$ gives

$$
T_{j}^{(0)} \geq T_{j}^{(1)} \geq \cdots \geq T_{j}^{(h)} .
$$

Then Clipper defines the contrast score of feature $j, j=1, \ldots, d$, in one of two ways:

$$
C_{j}:=\left\{\begin{array}{ll}
T_{j}^{(0)}-T_{j}^{(1)} & \text { if } T_{j}^{(0)}=T_{j}^{\sigma_{0}} \\
T_{j}^{(1)}-T_{j}^{(0)} & \text { otherwise }
\end{array} \quad\right. \text { minus contrast score }
$$

or

$$
C_{j}:=\left\{\begin{array}{ll}
\left|T_{j}^{(0)}\right| & \text { if } T_{j}^{(0)}=T_{j}^{\sigma_{0}}>T_{j}^{(1)} \\
0 & \text { if } T_{j}^{(0)}=T_{j}^{(1)} \\
-\left|T_{j}^{(0)}\right| & \text { otherwise }
\end{array} \quad \text { maximum contrast score } .\right.
$$

The intuition behind the contrast scores is that, if $C_{j}<0$, then $T_{j}^{(0)} \neq T_{j}^{\sigma_{0}}$, which means that at least one of $T_{j}^{\sigma_{1}}, \ldots, T_{j}^{\sigma_{h}}$ (calculated after random permutation) is greater than $T_{j}^{\sigma_{0}}$ calculated from the original data (identity permutation), suggesting that feature $j$ is likely an uninteresting feature in enrichment analysis. Figure 6b (right) shows a cartoon illustration of contrast scores when $m=2$ and $n=1$. Motivated by [64], we propose the following procedure for Clipper to control the FDR under the target level $q \in(0,1)$.

Definition 3 (Gimenez-Zou (GZ) procedure for thresholding contrast scores [64]) Given $h \in\left\{1, \cdots, h_{\max }\right\}$ and contrast scores $\left\{C_{j}\right\}_{j=1}^{d}, \mathcal{C}=\left\{\left|C_{j}\right|: C_{j} \neq 0 ; j=1, \ldots, d\right\}$ is defined as the set of non-zero absolute values of $C_{j}$ 's. The $G Z$ procedure finds a contrast-score cutoff $T^{G Z}$ based on the target FDR threshold $q \in(0,1)$ as:

$$
T^{G Z}:=\min \left\{t \in \mathcal{C}: \frac{\frac{1}{h}+\frac{1}{h} \operatorname{card}\left(\left\{j: C_{j} \leq-t\right\}\right)}{\operatorname{card}\left(\left\{j: C_{j} \geq t\right\}\right) \vee 1} \leq q\right\}
$$

and outputs $\left\{j: C_{j} \geq T^{G Z}\right\}$ as discoveries.

\section{Clipper: differential analysis with $m+n>2$}

For differential analysis, Clipper also uses permutation to construct contrast scores. When $m \neq n$, the equivalence classes of permutations are defined the same as for the enrichment analysis with $m \neq n$. When $m=n$, there is a slight change in the definition of equivalence classes of permutations: if $\sigma$ and $\sigma^{\prime}$ satisfy that

$$
\{\sigma(1), \cdots, \sigma(m)\}=\left\{\sigma^{\prime}(1), \cdots, \sigma^{\prime}(m)\right\} \text { or }\left\{\sigma^{\prime}(m+1), \cdots, \sigma^{\prime}(2 m)\right\},
$$

then we say that $\sigma$ and $\sigma^{\prime}$ are in one equivalence class. In total, there are $h_{\text {total }}:=\left(\begin{array}{c}m+n \\ m\end{array}\right)$ (when $m \neq n$ ) or $\left(\begin{array}{c}2 m \\ m\end{array}\right) / 2$ (when $m=n$ ) equivalence classes of permutations. Hence, to have more than one equivalence class, we cannot perform differential analysis with $m=n=1$; in other words, the total number of replicates $m+n$ must be at least 3 .

Then Clipper randomly samples $\sigma_{1}, \ldots, \sigma_{h}$ with equal probabilities without replacement from the $h_{\max }:=h_{\text {total }}-1$ equivalence classes that exclude the class containing $\sigma_{0}$, i.e., the identity permutation. Note that $h_{\max }$ is the maximum value $h$ can take. Next, Clipper computes $T_{j}^{\sigma_{\ell}}:=\left|t^{\text {minus }}\left(\boldsymbol{X}_{j}^{\sigma_{\ell}}, \boldsymbol{Y}_{j}^{\sigma_{\ell}}\right)\right|$, where $\boldsymbol{X}_{j}^{\sigma_{\ell}}$ and $\boldsymbol{Y}_{j}^{\sigma_{\ell}}$ are the permuted data defined in (9), and it defines $C_{j}$ as the contrast score of feature $j, j=1, \ldots, d$, in the same ways as 
in (10) or (11). Figure 6b (left) shows a cartoon illustration of contrast scores when $m=2$ and $n=1$.

Same as in the enrichment analysis with $m \neq n$, Clipper also uses the GZ procedure [64] to set a cutoff on contrast scores to control the FDR under the target level $q \in(0,1)$.

\section{Clipper: discussion on contrast scores}

Granted, when we use permutations to construct contrast scores in the GZ procedure, we can convert contrast scores into permutation-based $p$-values (see Additional File 1: Section S1.1.2). However, when the numbers of replicates are small, the number of possible permutations is small, so permutation-based $p$-values would have a low resolution (e.g., when $m=2$ and $n=1$, the number of non-identity permutations is only 2 ). Hence, applying the $\mathrm{BH}$ procedure to the permutation-based $p$-values would result in almost no power. Although Yekutieli and Benjamini proposed another thresholding procedure for permutation-based $p$-values [68], it still requires the number of permutations to be large to obtain a reliable FDR control. Furthermore, if we apply the SeqStep+ procedure by Barber and Candés [35] to permutation-based $p$-values, it would be equivalent to our application of the GZ procedure to contrast scores (Additional File 1: Section S1.1.2).

For both differential and enrichment analyses, the two contrast scores (minus and maximum) can both control the FDR. Based on the power comparison results in Additional File 1: Section S3 and Additional File 1: Figures S24-S28, Clipper has the following default choice of contrast score: for the enrichment analysis with $m=n$, Clipper uses the BC procedure with the minus contrast score; for the enrichment analysis with $m \neq n$ or the differential analysis, Clipper uses the GZ procedure with the maximum contrast score.

\section{Generic FDR control methods}

In our simulation analysis, we compared Clipper against generic FDR control methods including $p$-value-based methods and local-fdr-based methods. Briefly, each $p$-valuebased method is a combination of a $p$-value calculation approach and a $p$-value thresholding procedure. We use either the "paired" or "pooled" approach (see next paragraph) to calculate $p$-values of features and then threshold the $p$-values using the $\mathrm{BH}$ procedure (Additional File 1: Definition S1) or Storey's qvalue procedure (Additional File 1: Definition S2) to make discoveries (Additional File 1: Section S1.1). As a result, we have four $p$-value-based methods: BH-pair, BH-pool, qvalue-pair, and qvalue-pool (Fig. 1b).

Regarding the existing $p$-value calculation approaches in bioinformatics tools, we categorize them as "paired" or "pooled." The paired approach has been widely used to detect DEGs and protein-binding sites $[1,2,4,5]$. It examines one feature at a time and compares the feature's measurements between two conditions using a statistical test. In contrast, the pooled approach is popular in proteomics for identifying peptide sequences from MS data [69]. For every feature, it defines a test statistic and estimates a null distribution by pooling all features' observed test statistic values under the background condition. Finally, it calculates a $p$-value for every feature based on the feature's observed test statistic under the experimental condition and the null distribution.

In parallel to $p$-value-based methods, local-fdr-based methods estimate local fdrs of features and then threshold the local fdrs using the locfdr procedure (Additional File 1: Definition S5) to make discoveries. The estimation of local fdrs takes one of two approaches: (1) empirical null, which is estimated parametrically from the test statistic 
values that are likely drawn from the null distribution, and (2) swapping null, which is constructed by swapping measurements between experimental and background conditions. The resulting two local-fdr-based-methods are referred to as locfdr-emp and locfdr-swap (Figs. 1b and 2). Additional File 1: Section S1 provides a detailed explanation of these generic methods and how we implemented them in this work.

Specific to the $p$-value-based methods, for the paired approach, besides the ideal implementation that uses the correct model to calculate $p$-values (BH-pair-correct and qvalue-pair-correct), we also consider common mis-implementations. The first misimplementations is misspecification of the distribution (BH-pair-mis and qvalue-pairmis). An example is the detection of protein-binding sites from ChIP-seq data. A common assumption is that ChIP-seq read counts in a genomic region (i.e., a feature) follow the Poisson distribution [1,2], which implies that the counts have the variance equal to the mean. However, if only two replicates are available, it is impossible to check whether this Poisson distribution is reasonably specified. The second mis-implementation is the misspecification of a two-sample test as a one-sample test (BH-pair-2as1 and qvalue-pair2as1), which ignores the sampling randomness of replicates under one condition. This issue is implicit but widespread in bioinformatics methods $[1,70]$.

To summarize, we compared Clipper against the following implementations of generic FDR control methods:

- BH-pool or qvalue-pool: $p$-values calculated by the pooled approach and thresholded by the $B H$ or qvalue procedure.

- BH-pair-correct or qvalue-pair-correct: $p$-values calculated by the paired approach with the correct model specification and thresholded by the $B H$ or qvalue procedure.

- BH-pair-mis or qvalue-pair-mis: $p$-values calculated by the paired approach with a misspecified model and thresholded by the $B H$ or qvalue procedure.

- BH-pair-2as1 or qvalue-pair-2as1: $p$-values calculated by the paired approach that misformulates a two-sample test as a one-sample test (2as1) and thresholded by the $B H$ or qvalue procedure.

- locfdr-emp: local fdrs calculated by the empirical null approach and thresholded by the locfdr procedure.

- locfdr-swap: local fdrs calculated by the swapping approach and thresholded by the locfdr procedure.

\section{Software packages used in this study}

- p.adjust $\mathrm{R}$ function (in R package stat $\mathrm{s}$ v 4.0.2 with default arguments) [14]: used for BH-pool, BH-pair-correct, BH-pair-mis, and BH-pair-2as1.

- qvalue R package (v 2.20.0 with default arguments) [71]: used for qvalue-pool, qvalue-pair-correct, qvalue-pair-mis, and qvalue-pair-2as1.

- locfdr R package (v 1.1-8 with default arguments) [72]: used for locfdr-emp.

- MACS2 software package (v 2.2.6 with default settings) [1]: available at https:// github.com/macs3-project/MACS/releases/tag/v2.2.6.

- ChIPulate software package [45]: available at https://github.com/vishakad/chipulate.

- HOMER software package (findPeaks v 3.1.9.2 with default settings) [2]: available at https://www.bcgsc.ca/platform/bioinfo/software/findpeaks/releases/3.1.9.2/ findpeaks3-1-9-2-tar.gz. 
- SEQUEST in Proteome Discoverer (v 2.3.0.523 with default settings) [3]: commercial software by ThermoScientific.

- edgeR R package (v 3.30 .0 with default arguments) [4]: available at https://www. bioconductor.org/packages/release/bioc/html/edgeR.html.

- DESeq2 R package (v 1.28.1 with default arguments) [5]: available at https:// bioconductor.org/packages/release/bioc/html/DESeq2.html.

- limma R package (v 3.44.3 with default arguments) [10]: available at https://www. bioconductor.org/packages/release/bioc/html/limma.html.

- MAST R package (v 1.14.0 with default arguments) [57]: available at https://www. bioconductor.org/packages/release/bioc/html/MAST.html.

- monocle3 $\mathrm{R}$ package (v 0.2.3.0 with default arguments) [58]: available at https:// github.com/cole-trapnell-lab/monocle3.

- MultiHiCcompare R package (v 1.6.0 with default arguments) [11]: available at https://bioconductor.org/packages/release/bioc/html/multiHiCcompare.html.

- diffHic R package (v 1.20.0 with default arguments) [13]: available at https://www. bioconductor.org/packages/release/bioc/html/diffHic.html.

- FIND R package (v 0.99 with default arguments) [12]: available at https://bitbucket. org/nadhir/find/src/master/.

\title{
Supplementary Information
}

The online version contains supplementary material available at https://doi.org/10.1186/s13059-021-02506-9.

\begin{abstract}
Additional file 1: Supplementary materials. It includes a review of generic FDR control methods (Section S1 [73]), the detailed Clipper methodology (Section S2), the comparison of Clipper variant algorithms (Section S3), data generation and detailed implementation of the paired approach (a $p$-value calculation approach) in simulation studies (Section S4), bioinformatic methods with FDR control functionality (Section S5 [74, 75]), benchmark data generation in omics data applications (Section S6 [76-80]), implementation of Clipper in omics data applications (Section S7), proofs (Section S8), and Figures S1-S29.
\end{abstract}

Additional file 2: Supplementary table. Biological functions of the DEGs (between classical and non-classical human monocytes) that were identified by Clipper only but missed by both edgeR and DESeq2 from the human monocyte RNA-seq dataset (SRA accession number SRP082682).

Additional file 3: Review history

\section{Acknowledgements}

The authors would like to thank Dr. Yu-Cheng Yang for his suggestions on the figures and R package. The authors would also like to thank Mr. Nikos Ignatiadis, Dr. Lihua Lei, and Dr. Rina Barber for their insightful comments after we presented this work at the International Seminar on Selective Inference (https://www.selectiveinferenceseminar.com/past-talks). The authors also appreciate the comments and feedback from Mr. Tianyi Sun, Ms. Kexin Li, and other members of the Junction of Statistics and Biology at UCLA (http://jsb.ucla.edu).

\section{Peer review information}

Anahita Bishop was the primary editor of this article and managed its editorial process and peer review in collaboration with the rest of the editorial team.

\section{Review history}

The review history is available as Additional file 3.

\section{Authors' contributions}

J.J.L., X.G., and Y.E.C. developed the methodology. X.G. performed the simulation analysis and the DEG analysis on semi-synthetic single-cell RNA-seq data. X.G. did the peak calling analysis, advised by W.L.. Y.E.C. did the DEG analysis on semi-synthetic bulk RNA-seq data, the DIR analysis, and the peptide identification analysis. The real data used in peptide identification analysis was generated by M.M., K.W., and A.M., supported by L.D.W. X.G. and Y.E.C. performed DEG analysis on real bulk RNA-seq data, the results of which were interpreted by X.G., D.S., and N.W. X.G. and Y.E.C. developed the R package. Y.E.C. wrote the mathematical proof. X.G., Y.E.C., and J.J.L. wrote the manuscript. All authors read and approved the final manuscript.

\section{Funding}

This work was supported by the following grants: NIH-NCI T32LM012424 (to Y.E.C.); NCI K08 CA201591, the Gabrielle's Angel Foundation and Alex's Lemonade Stand Foundation (to L.D.W.); NIH R01HG007538, R01CA193466, and 
R01CA228140 (to W.L.); National Science Foundation DBI-1846216 and DMS-2113754, NIH/NIGMS R01GM120507 and R35GM140888, Johnson \& Johnson WiSTEM2D Award, Sloan Research Fellowship, and UCLA David Geffen School of Medicine W.M. Keck Foundation Junior Faculty Award (to J.J.L.).

\title{
Availability of data and materials
}

- The Clipper R package is available at https://github.com/JSB-UCLA/Clipper/ [81].

- The code and processed data for reproducing the figures are available at https://zenodo.org/record/5115468 (DOI number 10.5281/zenodo.5115468) [82].

- A video introduction of Clipper is available at https://youtu.be/-GXyHiJMpLo.

- Real datasets:

- The H3K4me3 ChIP-seq dataset with one experimental sample (GEO accession number GSM733708) and two control samples (GEO accession number GSM733742) from the cell line GM12878 is available at ftp://hgdownload.cse.ucsc.edu/goldenPath/hg19/encodeDCC/wgEncodeBroadHistone/, with the experimental sample wgEncodeBroadHistoneGm12878H3k4me3St dAlnRep1.bam and the two control samples wgEncodeBroadHistoneGm12878ControlStdAlnRep1.bam wgEncodeBroadHistoneGm12878ControlstdAlnRep2 . bam. The processed dataset is available at https://zenodo.org/record/5115468.

- The Pfu mass spectrometry proteomics data have been deposited to the ProteomeXchange Consortium via the PRIDE [83] partner repository with the dataset identifier PXD028558 (https://www.ebi.ac.uk/pride/ archive/projects/PXD028558).

The processed MS benchmark dataset is available as

data/pfu/Archaea Sequest DECOYS 100_FDR.xlsx for the decoy PSMs and data/pfu/Archaea Sequest Targets 100_FDR.xlsx for the target PSMs at https://zenodo.org/record/5202768.

- The human monocyte RNA-seq dataset (SRA accession number SRP082682) is available at https://www. ncbi.nlm.nih.gov/Traces/study/?acc=srp082682. The dataset includes 17 samples of classical monocytes and 17 samples of non-classical monocytes, and it is converted to a sample-by-gene count matrix by $\mathrm{R}$ package GenomicFeatures (v 1.40.1). The processed count matrix is available at https://zenodo.org/ record/5115468.

- The Hi-C dataset from the cell line GM12878 (GEO accession number GSE63525) is available at https:// www.ncbi.nlm.nih.gov/geo/query/acc.cgi?acc=GSE63525. The count matrix is under the filename GSE63525_GM12878_primary_intrachromosomal_contact_matrices.tar.gz, and the matrix corresponding to Chromosome 1 and bin width $1 \mathrm{MB}$ is used. The processed dataset is available at https://zenodo.org/record/5115468.

- The PBMC single-cell RNA-seq dataset (GEO accession number GSE132044) is available at https://www. ncbi.nlm.nih.gov/geo/query/acc.cgi?acc=GSE132044. The processed dataset is available at https://zenodo. org/record/5115468.

\section{Declarations}

\section{Ethics approval and consent to participate}

Not applicable.

\section{Competing interests}

L.D.W. holds equity in Magenta Therapeutics.

\begin{abstract}
Author details
${ }^{1}$ Department of Statistics, University of California, Los Angeles, 90095 CA, USA. ${ }^{2}$ Interdepartmental Program in Bioinformatics, University of California, Los Angeles, 90095 CA, USA. ${ }^{3}$ Beckman Research Institute, City of Hope National Medical Center, Duarte, 91010 CA, USA. ${ }^{4}$ The Quantitative and Computational Biology section, University of Southern California, Los Angeles, 90089 CA, USA. ${ }^{5}$ Division of Computational Biomedicine, Department of Biological Chemistry, School of Medicine, University of California, Irvine, 92697 CA, USA. ${ }^{6}$ Department of Human Genetics, University of California, Los Angeles, 90095 CA, USA. ${ }^{7}$ Department of Computational Medicine, University of California, Los Angeles, 90095 CA, USA. ${ }^{8}$ Department of Biostatistics, University of California, Los Angeles, 90095 CA, USA.
\end{abstract}

Received: 16 March 2021 Accepted: 21 September 2021

Published online: 11 October 2021

\section{References}

1. Zhang Y, LiuT, Meyer CA, Eeckhoute J, Johnson DS, Bernstein BE, Nusbaum C, Myers RM, Brown M, Li W, et al. Model-based analysis of chip-seq (macs). Genome Biol. 2008;9(9):1-9.

2. Heinz S, Benner C, Spann N, Bertolino E, Lin YC, Laslo P, Cheng JX, Murre C, Singh H, Glass CK. Simple combinations of lineage-determining transcription factors prime cis-regulatory elements required for macrophage and $b$ cell identities. Mol Cell. 2010;38(4):576-89.

3. Washburn MP, Wolters D, Yates JR. Large-scale analysis of the yeast proteome by multidimensional protein identification technology. Nat Biotechnol. 2001;19(3):242-7. 
4. Robinson MD, McCarthy DJ, Smyth GK. edger: a bioconductor package for differential expression analysis of digital gene expression data. Bioinformatics. 2010;26(1):139-40.

5. Love MI, Huber W, Anders S. Moderated estimation of fold change and dispersion for rna-seq data with deseq2. Genome Biol. 2014;15(12):550.

6. Trapnell $C$, Hendrickson DG, Sauvageau M, Goff $L$, Rinn $J$, Pachter $L$. Differential analysis of gene regulation at transcript resolution with rna-seq. Nat Biotechnol. 2013;31(1):46-53.

7. $\mathrm{Li}$ J, Witten DM, Johnstone IM, Tibshirani R. Normalization, testing, and false discovery rate estimation for rna-sequencing data. Biostatistics. 2012;13(3):523-38.

8. Hardcastle TJ, Kelly KA. bayseq: empirical bayesian methods for identifying differential expression in sequence count data. BMC Bioinforma. 2010;11(1):1-14.

9. Smyth G. Linear models and empirical bayes methods for assessing differential expression in microarray experiments. Stat Appl Genet Mol Biol. 2004;3(1). https://doi.org/10.2202/1544-6115.1027.

10. Ritchie ME, Phipson B, Wu D, Hu Y, Law CW, Shi W, Smyth GK. limma powers differential expression analyses for rna-sequencing and microarray studies. Nucleic acids research. 2015;43(7):e47.

11. Stansfield JC, Cresswell KG, Dozmorov MG. multihiccompare: joint normalization and comparative analysis of complex hi-c experiments. Bioinformatics. 2019;35(17):2916-23.

12. Djekidel MN, Chen Y, Zhang MQ. Find: differential chromatin interactions detection using a spatial poisson process. Genome Res. 2018;28(3):412-22.

13. Lun AT, Smyth GK. diffhic: a bioconductor package to detect differential genomic interactions in hi-c data. BMC Bioinforma. 2015;16(1):1-11.

14. Benjamini Y, Hochberg Y. Controlling the false discovery rate: a practical and powerful approach to multiple testing. J R Stat Soc Ser B Methodol. 1995;57(1):289-300.

15. Efron B, Tibshirani R. Empirical bayes methods and false discovery rates for microarrays. Genet Epidemiol. 2002;23(1): $70-86$.

16. Efron B, Tibshirani R, Storey JD, Tusher V. Empirical bayes analysis of a microarray experiment. J Am Stat Assoc. 2001;96(456):1151-60.

17. Stephens M. False discovery rates: a new deal. Biostatistics. 2017;18(2):275-94.

18. Storey JD, Tibshirani R. Statistical significance for genomewide studies. Proc Natl Acad Sci. 2003;100(16):9440-5.

19. Reiner A, Yekutieli $D$, Benjamini Y. Identifying differentially expressed genes using false discovery rate controlling procedures. Bioinformatics. 2003;19(3):368-75.

20. Yang B, Wu Y-J, Zhu M, Fan S-B, Lin J, Zhang K, Li S, Chi H, Li Y-X, Chen H-F, et al. Identification of cross-linked peptides from complex samples. Nat Methods. 2012;9(9):904-6.

21. White JR, Nagarajan N, Pop M. Statistical methods for detecting differentially abundant features in clinical metagenomic samples. PLoS Comput Biol. 2009;5(4):e1000352.

22. Shabalin AA. Matrix eqtl: ultra fast eqtl analysis via large matrix operations. Bioinformatics. 2012;28(10):1353-8.

23. Hawinkel S, Mattiello F, Bijnens $L$, Thas $O$. A broken promise: microbiome differential abundance methods do not control the false discovery rate. Brief Bioinform. 2019;20(1):210-21.

24. Zheng $Y$, Keleş $\mathrm{S}$. Freehi-c simulates high-fidelity hi-c data for benchmarking and data augmentation. Nat Methods. 2020;17(1):37-40.

25. Storey JD. A direct approach to false discovery rates. J R Stat Soc Ser B Stat Methodol. 2002;64(3):479-98.

26. Benjamini Y, Hochberg Y. Multiple hypotheses testing with weights. Scand J Stat. 1997;24(3):407-18.

27. Ignatiadis N, Klaus B, Zaugg JB, Huber W. Data-driven hypothesis weighting increases detection power in genome-scale multiple testing. Nat Methods. 2016;13(7):577-80.

28. Lei L, Fithian W. AdaPT: an interactive procedure for multiple testing with side information. J R Stat Soc Ser B Stat Method. 2018;80(4):649-79.

29. Boca SM, Leek JT. A direct approach to estimating false discovery rates conditional on covariates. PeerJ. 2018;6:e6035.

30. Ho J, Tumkaya T, Aryal S, Choi H, Claridge-Chang A. Moving beyond $\mathrm{p}$ values: data analysis with estimation graphics. Nat Methods. 2019;16(7):565-6.

31. Song D, Li JJ. PseudotimeDE: inference of differential gene expression along cell pseudotime with well-calibrated p-values from single-cell RNA sequencing data. Genome Biol. 2021;22:124.

32. Tusher VG, Tibshirani $R$, Chu G. Significance analysis of microarrays applied to the ionizing radiation response. Proc Natl Acad Sci. 2001;98(9):5116-21.

33. Hemerik J, Goeman JJ. False discovery proportion estimation by permutations: confidence for significance analysis of microarrays. J R Stat Soc Ser B Stat Methodol. 2018;80(1):137-55.

34. Hemerik J, Solari A, Goeman JJ. Permutation-based simultaneous confidence bounds for the false discovery proportion. Biometrika. 2019;106(3):635-49.

35. Barber RF, Candès EJ. Controlling the false discovery rate via knockoffs. Ann Stat. 2015;43(5):2055-85.

36. Arias-Castro E, Chen S. Distribution-free multiple testing. Electron J Stat. 2017;11(1):1983-2001.

37. Benjamini Y. Selective inference: The silent killer of replicability. Harv Data Sci Rev. 2020;2(4). https://doi.org/10.1162/ 99608f92.fc62b261.

38. Emery K, Hasam S, Noble WS, Keich U. Multiple competition-based FDR control and its application to peptide detection. In: Schwartz R, editor. Research in Computational Molecular Biology. RECOMB 2020. Lecture Notes in Computer Science, vol 12074. Cham: Springer; 2020. p. 54-71.

39. Sarkar A, Stephens M. Separating measurement and expression models clarifies confusion in single cell RNA-sequencing analysis. Nat Genet. 2021;53(6):770-7.

40. Park PJ. Chip-seq: advantages and challenges of a maturing technology. Nat Rev Genet. 2009;10(10):669-80.

41. Mitchell PJ, Tjian R. Transcriptional regulation in mammalian cells by sequence-specific dna binding proteins. Science. 1989;245(4916):371-8.

42. Ptashne M, Gann A. Transcriptional activation by recruitment. Nature. 1997;386(6625):569-77.

43. Bailey T, Krajewski P, Ladunga I, Lefebvre C, Li Q, Liu T, Madrigal P, Taslim C, Zhang J. Practical guidelines for the comprehensive analysis of chip-seq data. PLoS Comput Biol. 2013;9(e1003326):. 
44. ENCODE Project Consortium, et al. An integrated encyclopedia of dna elements in the human genome. Nature. 2012;489(7414):57-74.

45. Datta V, Hannenhalli S, Siddharthan R. Chipulate: A comprehensive chip-seq simulation pipeline. PLoS Comput Biol. 2019;15(3):e1006921.

46. Diaz A, Park K, Lim D, Song JS. Normalization, bias correction, and peak calling for ChIP-seq. Stat Appl Genet Mol Biol. 2012;11(3). https://doi.org/10.1515/1544-6115.1750.

47. Bogdanow B, Zauber $H$, Selbach M. Systematic errors in peptide and protein identification and quantification by modified peptides. Mol Cell Proteomics. 2016;15(8):2791-801.

48. Perkins D, Pappin D, Creasy D, Cottrell J. Probability-based protein identification by searching sequence databases using mass spectrometry data. Electrophor Int J. 1999;20:3551-67.

49. Bern M, Kil Y, Becker C. Byonic: advanced peptide and protein identification software. Curr Protoc Bioinforma. 2012;40(1):13-20.

50. Robinson M, Oshlack A. A scaling normalization method for differential expression analysis of rna-seq data. Genome Biol. 2010;11(3):1-9.

51. Williams C, Baccarella A, Parrish J, Kim C. Empirical assessment of analysis workflows for differential expression analysis of human samples using rna-seq. BMC Bioinforma. 2017;18(1):38.

52. Gierliński M, Cole C, Schofield P, Schurch N, Sherstnev A, Singh V, Wrobel N, Gharbi K, Simpson G, Owen-Hughes $T$, et al. Statistical models for rna-seq data derived from a two-condition 48-replicate experiment. Bioinformatics. 2015;31:3625-30.

53. Korthauer $K$, Kimes $P$, Duvallet $C$, Reyes A, Subramanian A, Teng M, Shukla C, Alm E, Hicks S. A practical guide to methods controlling false discoveries in computational biology. Genome Biol. 2019;20(1):1-21.

54. Li Y, GeX, Peng F, Li W, Li JJ. A large-sample crisis? Exaggerated false positives by popular differential expression methods. bioRxiv 2021.08.25.457733. https://doi.org/10.1101/2021.08.25.457733.

55. Li Q, Brown J, Huang H, Bickel P. Measuring reproducibility of high-throughput experiments. Ann Appl Stat. 2011;5(3):1752-79.

56. Yu G, Wang L-G, Han Y, He Q-Y. clusterprofiler: an r package for comparing biological themes among gene clusters. Omics J Integr Biol. 2012;16(5):284-7.

57. Finak G, McDavid A, Yajima M, Deng J, Gersuk V, Shalek A, Slichter C, Miller H, McElrath MJ, Prlic M, et al. Mast: a flexible statistical framework for assessing transcriptional changes and characterizing heterogeneity in single-cell rna sequencing data. Genome Biol. 2015;16(1):1-13.

58. Qiu X, Hill A, Packer J, Lin D, Ma Y-A, Trapnell C. Single-cell mrna quantification and differential analysis with census. Nat Methods. 2017;14(3):309-15.

59. Soneson C, Robinson M. Bias, robustness and scalability in single-cell differential expression analysis. Nat methods. 2018;15(4):255.

60. Sun T, Song D, Li WV, Li JJ. scdesignz: an interpretable simulator that generates high-fidelity single-cell gene expression count data with gene correlations captured. bioRxiv. 2020.

61. Ding J, Adiconis X, Simmons S, Kowalczyk MS, Hession CC, Marjanovic ND, Hughes T, Wadsworth M, Burks T, Nguyen LT, et al. Systematic comparison of single-cell and single-nucleus ma-sequencing methods. Nat Biotechnol. 2020;38(6):737-46

62. Zheng G, Terry J, Belgrader P, Ryvkin P, Bent Z, Wilson R, Ziraldo S, Wheeler T, McDermott G, Zhu J, et al. Massively parallel digital transcriptional profiling of single cells. Nat Commun. 2017;8(1):1-12.

63. Macosko E, Basu A, Satija R, Nemesh J, Shekhar K, Goldman M, Tirosh I, Bialas A, Kamitaki N, Martersteck E, et al. Highly parallel genome-wide expression profiling of individual cells using nanoliter droplets. Cell. 2015;161(5): 1202-14.

64. Gimenez JR, Zou J. Improving the stability of the knockoff procedure: Multiple simultaneous knockoffs and entropy maximization. 22nd Int Conf Artif Intell Stat PMLR. 2019;89:2184-92.

65. Wang N, Lefaudeux D, Mazumder A, Li JJ, Hoffmann A. Identifying the combinatorial control of signal-dependent transcription factors. PLOS Comput Biol. 2021;17(6):e1009095.

66. Thorsen J, Brejnrod A, Mortensen M, Rasmussen M, Stokholm J, Al-Soud WA, Sørensen S, Bisgaard H, Waage J. Large-scale benchmarking reveals false discoveries and count transformation sensitivity in $16 \mathrm{~s}$ rrna gene amplicon data analysis methods used in microbiome studies. Microbiome. 2016;4(1):62.

67. He K, Li M, Fu Y, Gong F, Sun X. Null-free false discovery rate control using decoy permutations for multiple testing. arXiv preprint arXiv:1804.08222. 2018.

68. Yekutieli D, Benjamini Y. Resampling-based false discovery rate controlling multiple test procedures for correlated test statistics. J Stat Plan Infer. 1999;82(1-2):171-96.

69. Nesvizhskii A. A survey of computational methods and error rate estimation procedures for peptide and protein identification in shotgun proteomics. J proteomics. 2010;73(11):2092-123.

70. Li W, Xu H, Xiao T, Cong L, Love MI, Zhang F, Irizarry RA, Liu JS, Brown M, Liu XS. Mageck enables robust identification of essential genes from genome-scale crispr/cas9 knockout screens. Genome Biol. 2014;15(12):554.

71. Storey J, Bass A, Dabney A, Robinson David. qvalue: Q-value estimation for false discovery rate control. 2020. https://github.com/jdstorey/qvalue. R package version 2.20.0. Accessed 11 July 2021.

72. Efron B. Large-scale simultaneous hypothesis testing: the choice of a null hypothesis. J Am Stat Assoc. 2004;99(465): 96-104.

73. Barber RF, Candès E, et al. A knockoff filter for high-dimensional selective inference. Ann Stat. 2019;47(5):2504-37.

74. Spivak M, Weston J, Bottou L, Käll L, Noble WS. Improvements to the percolator algorithm for peptide identification from shotgun proteomics data sets. J Proteome Res. 2009;8(7):3737-45.

75. Stansfield J, Cresswell K, Vladimirov V, Dozmorov M. Hiccompare: an r-package for joint normalization and comparison of hi-c datasets. BMC Bioinforma. 2018;19(1):279.

76. Howe KL, Achuthan P, Allen J, Allen J, Alvarez-Jarreta J, Amode MR, Armean IM, Azov AG, Bennett R, Bhai J, et al. Ensembl 2021. Nucleic Acids Res. 2021;49(D1):D884-91. 
77. Mellacheruvu D, Wright Z, Couzens A, Lambert J-P, St-Denis N, Li T, Miteva Y, Hauri S, Sardiu M, Low TY, et al. The crapome: a contaminant repository for affinity purification-mass spectrometry data. Nat Methods. 2013;10(8):730-6.

78. Cox J, Mann M. Maxquant enables high peptide identification rates, individualized ppb-range mass accuracies and proteome-wide protein quantification. Nat Biotechnol. 2008;26(12):1367-72.

79. Goloborodko AA, Levitsky LI, Ivanov MV, Gorshkov MV. Pyteomics - a python framework for exploratory data analysis and rapid software prototyping in proteomics. J Am Soc Mass Spectrom. 2013;24(2):301-4.

80. Levitsky L, Klein J, Ivanov M, Gorshkov M. Pyteomics 4.0: five years of development of a python proteomics framework. J Proteome Res. 2018;18(2):709-14.

81. Ge X, Chen Y, Li JJ. Clipper: p-value-free FDR control on high-throughput data from two conditions. https://github. com/JSB-UCLA/Clipper. R package version 0.0.0.9. Accessed 11 July 2021.

82. Ge X, Chen Y, Li JJ. Clipper: p-value-free FDR control on high-throughput data from two conditions. 2021. https:// zenodo.org/record/5115468.

83. Perez-Riverol Y, Csordas A, Bai J, Bernal-Llinares M, Hewapathirana S, Kundu D, Inuganti A, Griss J, Mayer G, Eisenacher M, et al. The pride database and related tools and resources in 2019: improving support for quantification data. Nucleic Acids Res. 2019;47(D1):D442-50.

\section{Publisher's Note}

Springer Nature remains neutral with regard to jurisdictional claims in published maps and institutional affiliations.

- fast, convenient online submission

- thorough peer review by experienced researchers in your field

- rapid publication on acceptance

- support for research data, including large and complex data types

- gold Open Access which fosters wider collaboration and increased citations

- maximum visibility for your research: over $100 \mathrm{M}$ website views per year

At $\mathrm{BMC}$, research is always in progress.

Learn more biomedcentral.com/submissions 